\title{
MÉTODOS DE CONTROLE DE PROCESSO ON-LINE PARA ATRIBUTOS
}

\author{
Ana Cristina Vieira Belem
}

DISSERTAÇÃO APRESENTADA

$\mathrm{AO}$

INSTITUTO DE MATEMÁTICA E ESTATÍSTICA

DA

UNIVERSIDADE DE SÃO PAULO

PARA OBTENÇÃO DO GRAU

DE

MESTRE EM ESTATÍSTICA

Área de Concentração: Estatística

Orientador: Prof. Dr. Wagner Borges

- São Paulo, maio de 2001 - 


\title{
MÉTODOS DE CONTROLE DE PROCESSO ON-LINE PARA ATRIBUTOS
}

\author{
Este exemplar corresponde à redação final \\ da dissertação devidamente corrigida e \\ defendida por Ana Cristina Vieira Belem \\ e aprovada pela comissão julgadora.
}

São Paulo, 15 maio de 2001

Banca Examinadora:

- Prof. Dr. Wagner de Souza Borges (Orientador) - IME - USP

- Prof. Dr. Adilson Simonis - IME - USP

- Profa. Dra. Linda Lee Ho - EP - USP 
A meus queridos pais Humberto e Luisa, irmãos Júnior e Dirceu e meu futuro marido Renan. Vocês são a razão de minha existência. 


\section{Agradecimentos}

Gostaria de registrar meus agradecimentos às pessoas que contribuíram na execução deste trabalho.

Em primeiro lugar, agradeço ao Prof. Dr. Wagner Borges que me orientou dispensando enorme dedicação e apoio.

Aos amigos que estiveram sempre presentes. Em especial a Cláudia Daré, pela grande amizade e companherismo. Lilian e Roberto Quinino, pelo incentivo inicial. Aos colegas Aldy, Deusélio e Delhy, por todo apoio. Aos queridos amigos Marco, Myléne e Aurimar, por terem se tornado minha família enquanto estive em São Paulo.

Também agradeço aqueles que, mesmo de longe, me incentivaram e compreenderam minha constante ausência. As minhas avós Gaby, por suas orações, e Nelly, onde quer que esteja, sei que está junto de nós. Aos meus parentes e futuros parentes, por me fazerem sentir querida.

Gostaria, principalmente, de agradecer a meus pais que sempre me incentivaram e que, com dedicação e amor profundo, fizeram com que meu caminho fosse mais suave. Ao meu querido Renan, por todo amor, compreensão e por me encorajar nos momentos difíceis. A eles não tenho como expressar minha gratidão.

E finalmente agradeço a Deus, por ter me oferecido a oportunidade de percorrer esse caminho. 


\section{Resumo}

Uma classe de procedimentos utilizados para monitorar processos por atributos são os procedimentos introduzidos por Taguchi $(1981,1984,1985)$ e Taguchi et. al. (1989). Esses procedimentos envolvem apenas a inspeção de um item retirado da linha de produção, a cada intervalo fixo de $m$ itens produzidos, e o seu planejamento é tratado por Taguchi e seus colaboradores através de critérios econômicos.

Este trabalho tem como objetivo apresentar um modelo formal, unificado, para descrever o procedimento de Taguchi para atributos e obter, a partir dele, a expressão analítica para o custo médio assintótico do procedimento de controle, por item produzido. Neste contexto, o trabalho corrige um resultado obtido por de Nayebpour e Woodall (1993), no desenvolvimento de uma modelagem alternativa para o mecanismo de falha, quando este é monitorado pelo procedimento de Taguchi. Aborda, ainda, a questão da estimação dos parâmetros do processo a partir dos dados observados no procedimento de controle. Neste último caso, dá-se ênfase, em particular, ao ponto de mudança ou instante da falha do processo. 


\section{Abstract}

An important class of process monitoring procedures used to monitor processes for attributes are the procedures introduced by Taguchi $(1981,1984,1985)$ and Taguchi et. al. (1989). These procedures involve the inspection of an item out of every $m$ produced and their design is treated by Taguchi and his coauthors under an economical perspective.

The objective of this work is to present a formal unified model to describe Taguchi's monitoring procedures for attributes and to obtain, from it, the analytical expression for the asymptotic mean cost of the control procedure, per item produced. In this context, the correction of a result obtained by Nayebpour and Woodall (1993) in the development of an alternative model for the process failure mechanism, and the development of estimate in procedures parameters from observed process and control data, are present. In the lather case, emphasis is givem to the process change point. 


\section{Índice}

1 Controle Estatístico de Processos 2

2 Métodos de Controle de Taguchi para Atributos com Diagnóstico Perfeito $\mathbf{1 3}$

2.1 Abordagem de Taguchi para o Caso I . . . . . . . . . . . . . . . . . . . 14

2.2 Abordagem de Taguchi para o Caso I I. . . . . . . . . . . . . . . 20

2.3 Comentários sobre a Abordagem de Taguchi . . . . . . . . . . . . . 24

3 A Correção de Nayebpour and Woodall 27

$\begin{array}{ll}\text { Conclusão } & \mathbf{4 0}\end{array}$

$\begin{array}{ll}\text { Referências Bibliográficas } & 41\end{array}$ 


\section{Capítulo 1}

\section{Controle Estatístico de Processos}

Por maior que seja o esforço destinado ao desenvolvimento e a operação dos processos industriais, as características de qualidade do produto final estão sujeitas as variações resultantes de diversos fatores. Assim, se não houver interferência sobre os processos, grandes desvios nas características de qualidade dos produtos podem ser gerados, resultando em índices elevados de não conformidades.

O Controle Estatístico de Processo (CEP) é uma disciplina de gestão em que interferências sobre os processos são decididas com base na análise estatística de dados gerados por este. Neste contexto, uma ferramenta importante de análise é a carta de controle. Introduzidas por Shewhart nos anos 20, as cartas de controle têm como objetivo monitorar a estabilidade de características específicas do processo e sinalizar desvios em relação à condição estável, produzidos por diversos fatores ou causas especiais.

Quando a característica de interesse é a fração defeituosa do processo, a carta de controle normalmente utilizada é a cartas-p ou $n p$. Sua operação consiste em: coletar periodicamente amostras de itens produzidos; classificar cada item amostrado como conforme ou não conforme; nas cartas-p, constitui-se um gráfico formado pelos pontos $\left(j, p_{j}\right)$, em que $j$ indica a amostra observada e $p_{j}$ a fração defeituosa na mesma, $j \geq 1$. Esse gráfico contém, ainda, três retas auxiliares denominadas limite inferior de controle (LIC), linha central (LC) e limite superior de controle (LSC). O processo é declarado fora de controle se um ponto do gráfico é registrado acima do LSC ou abaixo do LIC. Outros critérios de intervenção podem ser definidos para aumentar a sensibilidade da carta de controle. Os mais comuns são: sequiência crescente ou decrescente de sete ou mais pontos consecutivos, seqüência de nove 
pontos consecutivos do mesmo lado da linha central e quatorze pontos consecutivos alternando acima e abaixo da linha central, conforme sugerido no Western Eletric Handbook (1956). Uma discussão a respeito de critérios de intervenção pode ser encontrada em Nelson (1984).

Nas cartas- $p$, a linha central é dada por $p_{0}$ e os limites de controle por

$$
p_{0} \pm L \sqrt{\frac{p_{0}\left(1-p_{0}\right)}{n}}
$$

em que $p_{0}$ é a proporção de itens defeituosos produzidos pelo processo em seu estado de controle, $n$ o tamanho da amostra e $L$ é o parâmetro que determina a distância dos limites de controle à linha central. Se as amostras não possuem o mesmo tamanho, $n$ é substituído por $n_{j}$ em (1.1), que representa o tamanho da amostra $j, j \geq 1$.

No estado de controle, supõe-se que os itens são produzidos independentemente com probabilidade $p_{0}$ de serem não conformes. Assim, se a fração defeituosa do processo, $p_{0}$, é desconhecida, é necessário estimá-la através de dados observados com o processo em condição de controle. O procedimento usual é selecionar $m$ amostras preliminares (geralmente, $m=20$ ou 25) e estimar $p_{0}$ por

$$
\hat{p}_{0}=\frac{\sum_{i=1}^{m} D_{j}}{m n} .
$$

em (1.2) $D_{j}$ denota o número de itens não conformes na amostra $j, 1 \leq j \leq m$. Os limites de controle são obtidos substituindo $p_{0}$ por $\hat{p}_{0}$ na expressão (1.1). Supõe-se ainda que desvios na fração defeituosa do processo ocorram apenas entre as janelas de "tempo" em que as amostras são obtidas. Dessa forma, $D_{j}, j \geq 1$, terá sempre distribuição binomial com parâmetros $n_{j}$ e $p_{j}$, em que $p_{j}$ é a fração defeituosa do processo durante a $j$-ésima janela de "tempo". Esta suposição corresponde à noção prática de formação de subgrupos racionais introduzida por Shewhart. Observe que, operar a carta de controle é equivalente a realizar, a cada amostra coletada, um teste de hipótese para a proporção não conforme do processo, em que a hipótese nula é $p_{j}=p_{0}$ (hipótese de controle) contra a alternativa $p_{j} \neq p_{0}$ (hipótese de perda de controle). 
O Exemplo 1.1 adaptado de Montgomery (1996) para ilustrar uma aplicação da carta$p$.

Exemplo 1.1: Uma Empresa fabricante de suco concentrado de laranja, está interessada em melhorar a qualidade de suas embalagens. O suco é envasado em um único tipo embalagem, com corpo de papelão e fundo de metal. A embalagem é produzida por uma máquina, que dobra e sela a parte de papelão e a coloca sobre o painel de metal. A inspeção das embalagens determina se, quando cheias, haverá vazamento. Isso poderá ocorrer se houver defeito de selagem, tanto na emenda do corpo de papelão quanto ao redor do fundo de metal. Assim, é de interesse construir uma carta de controle para monitorar a fração de embalagens não conformes produzidas pela máquina e verificar se as ações para reduzi-la são eficazes. Para determinar os limites da carta de controle, 30 amostras de 50 embalagens foram selecionadas a cada 30 minutos, em um período em que a máquina estava em operação contínua. Os dados são apresentados na Tabela 1.1.

Inicialmente, foi construída uma carta de controle preliminar para verificar se seria razoável supor que processo estava sob controle quando os dados foram coletados. Usando $L=3$ e $\hat{p}_{0}=0,2313$ para estimar a verdadeira fração defeituosa do processo, obteve-se LSC $=$ $0,2313+0,1789=0,4102$ e LIC $=0,2313-0,1789=0,0524$. A carta de controle é apresentada na Figura 1.1. Observe que dois pontos, referentes as amostras 15 e 23, superam o LSC, indicando que o processo não estava sob controle.

Investigando o processo, verificou-se que uma nova batelada de cartão foi colocada na produção durante um período de 30 minutos, justamente quando foi retirada a amostra 15. Acredita-se que, a introdução da nova batelada de matéria prima pode causar um desempenho irregular de produção. Além disso, um operador relativamente inexperiente assumiu temporariamente o controle da máquina durante o período no qual a amostra 23 foi retirada, ocasionando uma alta fração defeituosa. Consequentemente, as amostras 15 e 23 foram eliminadas e novos limites de controle foram calculados, obtendo-se LSC =0,3893 e LIC = 0,0407. A carta de controle com os limites revisados, sem os pontos 15 e 23, está apresentada na Figura 1.2. Observe agora, que nenhuma amostra excede os limite de controle. Assim, os limites da carta da Figura 1.2 foram usados para monitorar amostras futuras, concluindo-se a fase de estimação da fração defeituosa do processo e obtenção dos limites de controle. 
Tabela 1.1: Fração de embalagens não conformes.

\begin{tabular}{|c|c|c|}
\hline $\begin{array}{l}\text { Número } \\
\text { da } \\
\text { Amostra }\end{array}$ & $\begin{array}{c}\text { Número de } \\
\text { embalagens não } \\
\text { conformes }\left(D_{i}\right)\end{array}$ & $\begin{array}{c}\text { Fração não } \\
\text { conforme na } \\
\text { amostra }\left(\hat{p}_{i}\right)\end{array}$ \\
\hline 1 & 12 & 0.24 \\
\hline 2 & 15 & 0.30 \\
\hline 3 & 8 & 0.16 \\
\hline 4 & 10 & 0,20 \\
\hline 5 & 4 & 0.08 \\
\hline 6 & 7 & 0.14 \\
\hline 7 & 16 & 0.32 \\
\hline 8 & 9 & 0.18 \\
\hline 9 & 14 & 0.28 \\
\hline 10 & 10 & 0.20 \\
\hline 11 & 5 & 0.10 \\
\hline 12 & 6 & 0.12 \\
\hline 13 & 17 & 0.34 \\
\hline 14 & 12 & 0.24 \\
\hline 15 & 22 & 0.44 \\
\hline 16 & 8 & 0.16 \\
\hline 17 & 10 & 0.20 \\
\hline 18 & 5 & 0.10 \\
\hline 19 & 13 & 0.26 \\
\hline 20 & 11 & 0.22 \\
\hline 21 & 19 & 0.38 \\
\hline 22 & 18 & 0.36 \\
\hline 23 & 24 & 0.48 \\
\hline 24 & 15 & 0.30 \\
\hline 25 & 9 & 0.18 \\
\hline 26 & 12 & 0.24 \\
\hline 27 & 7 & 0.14 \\
\hline 28 & 13 & 0.26 \\
\hline 29 & 9 & 0.18 \\
\hline 30 & 6 & 0,12 \\
\hline Total & 347 & $\hat{p}_{0}=0,2313$ \\
\hline
\end{tabular}




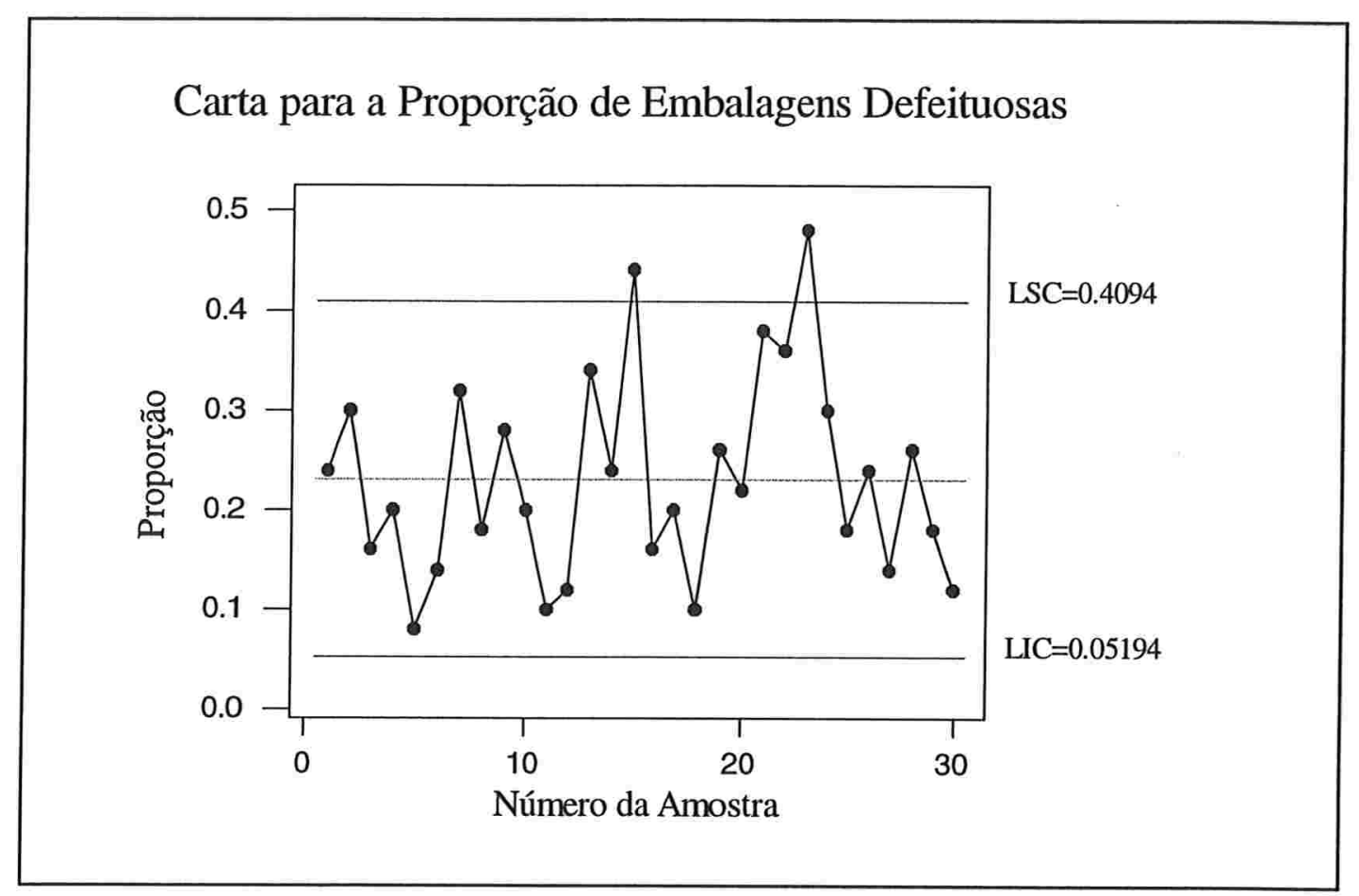

Figura 1.1: Carta de Controle para os dados da Tabela 1.1.

Carta para a Proporção de Embalagens Defeituosas

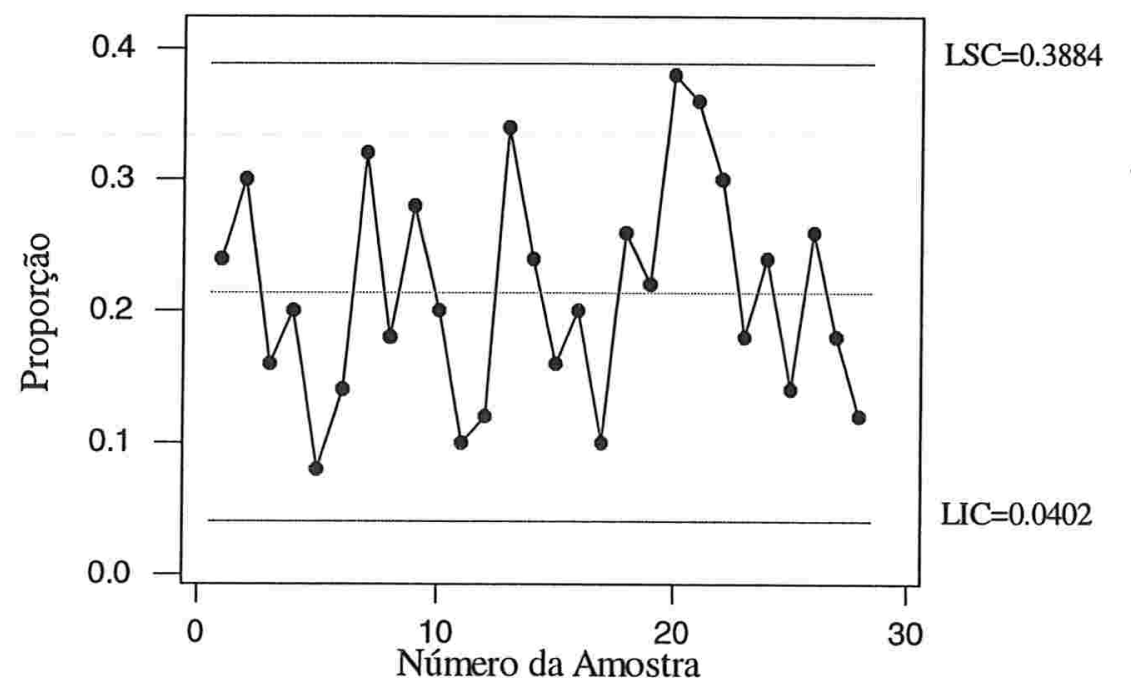


Figura 1.2: Carta de Controle para os dados da Tabela 1.1 sem as amostras 15 e 23.

No exemplo 1.1, o procedimento de controle utilizou amostras de tamanho $n=50$ selecionadas a cada 30 minutos e $L=3$. Que critérios foram utilizados na escolha desses parâmetros, isto é, no planejamento da carta de controle? Existem duas formas de tratar formalmente o planejamento destas cartas: através de critérios estatísticos de desempenho e de através de critérios econômicos de desempenho. Mais recentemente, têm sido desenvolvidos alguns trabalhos que envolvem critérios econômicos e estatísticos. Na prática, costuma-se a tomar $L=3$, que caracterizam os chamados limites de controle 3 sigma. A justificativa para o uso de $L=3$ é apenas histórica. Alterações no valor de $L$, entretanto, têm impacto direto nas probabilidades de alarme falso ou erro tipo $I$ (rejeitar a condição de controle quando ela é verdadeira) e de não-detecção ou erro tipo II (não rejeitar a condição de controle quando ela é falsa).

Vários critérios estatísticos têm sido sugeridos na literatura para determinar o tamanho $n$ e a freqüência de amostragem, $h$, para cartas-p (ou $n p$ ). Duncan (1986) sugere que o tamanho da amostra seja suficientemente grande para que, a probabilidade de detectar uma mudança de magnitude $\delta$ fixada, seja pelo menos 0.50 . Pela simetria da distribuição normal, $n$ deve ser tal que o limite superior de controle coincida exatamente com a fração não conforme que deseja-se detectar, ou seja, $\delta=3 \sqrt{\frac{p(1-p)}{n}} \leftrightarrow n=\left(\frac{3}{\delta}\right)^{2} p(1-p)$. Costuma-se sugerir também, que $n$ seja tal que a carta de controle tenha um limite inferior de controle positivo, ou seja, LIC $=p-3 \sqrt{\frac{p(1-p)}{n}}>0 \leftrightarrow n>9 \frac{(1-p)}{p}$. Este critério assegura a identificação de oportunidades de melhoria no processo. O tamanho da amostra também pode ser determinado com o auxílio das curvas características de operação (CCO). Estas curvas descrevem a probabilidade do erro tipo II em função da fração defeituosa do processo; provêm uma medida da sensibilidade da carta de controle em detectar mudanças nessa característica do processo. A probabilidade do erro tipo II de uma carta-p é definida por

$$
\beta(p)=P\{\hat{p} \leq L S C \mid p\}-P\{\hat{p}<L I C \mid p\}=P\left\{D_{j} \leq n L S C \mid p\right\}-P\left\{D_{j}<n L I C \mid p\right\},
$$


em que $D_{j}$ tem distribuição binomial com parâmetros $n$ e $p$ e $j$ é fixo. A escolha do tamanho da amostra é feita fixando-se a magnitude da mudança que se deseja detectar e o risco (probabilidade do erro tipo II) que se deseja correr.

Outra ferramenta de planejamento estatístico de cartas de controle, que também prevê uma medida do desempenho da carta, é a curva do comprimento médio da seqüência (CCMS). Esta curva descreve o número médio de pontos que serão marcados na carta de controle, até que um ponto seja marcado na região fora dos limites de controle, em função da fração defeituosa do processo. Especificamente

$$
\operatorname{CCMS}(p)=\frac{1}{1-\beta(p)}
$$

Vários autores tratam e sugerem soluções para a questão do planejamento estatístico das cartas carta-p (ou $n p$ ). Em particular: Burr (1953) propõe $n p_{0}>1$ e $L=3$; Cowden (1957) $n p_{0}>25$ e $L=3$; Feigenbaum (1961) sugere $n=25, h=1$ ou 8 horas e $L=3$, Juran et al. (1974) $n>50$ com $n p_{0}>4$ e $L=3$, Ishikawa (1976) $n>50$ com $3<n p_{0}<4, h=8$ horas e $L=3$ e Grant and Leavenworth (1980) propõe inspeção $100 \%, h=8$ horas e $L=3$. As propostas de maneira geral baseiam-se em critérios distintos, embora todos concordem em recomendar a escolha de $L=3$.

O primeiro a sugerir critérios econômicos para o planejamento de cartas de controle foi Ducan (1956). Para cartas-p, especificamente, a primeira abordagem em planejamento econômico foi proposta por Ladany (1973) que desenvolveu um modelo admitindo a ocorrência de somente um tipo de causa especial. Após o trabalho de Ladany, diversos autores fizeram contribuições importantes no planejamento econômico de cartas-p. Em particular, Chiu (1975) que modificou o trabalho de Duncan para cartas-np, supondo ainda que, após a detecção de um desvio em seu estado de controle, o processo é interrompido até ser ajustado. Montgomery, Heikes e Mance (1975) desenvolveram um modelo para a carta $n p$, em que o processo está sujeito à ocorrência de múltiplas causas especiais. Esta flexibilidade também é incorporada por Chiu (1976) em seu modelo proposto em 1975 [Chiu (1975)]. Gibra (1978) desenvolve dois modelos diferentes para o planejamento econômico de cartas-np; o primeiro, supõe que o processo continua em operação durante a investigação da causa especial; e o segundo, supõe que o processo é interrompido. A abordagem de Gibra é similar a Duncan 
(1956) mas incorpora outros fatores de custo que podem ocorrem na prática. Em outra direção Montgomery e Heikes (1976) avaliaram o impacto do principal elemento dos modelos de planejamento econômico do procedimento de controle: a distribuição do tempo em que o processo permanece sob controle, denominada Mecanismo do Processo de Falha (MPF). Montgomery e Heikes apresentaram exemplos numéricos para cartas-np supondo MPF's poissonianos, geométricos e logarítmicos. Duncan (1978) apresentou uma análise extensiva da sensibilidade das cartas- $p$ planejadas economicamente, em processos sujeitos a somente uma causa especial. Willians, Looney and Peters (1985), por sua vez, desenvolveram planos amostrais para o planejamento de cartas-np. De maneira geral, as seguintes suposições sobre o processo de produção são feitas no planejamento econômico de cartas-p:

1. processo em seu estado de controle, produz uma fração constante, $p_{0}$, de itens não conformes;

2. Após um período de tempo $\tau$, ocorre uma causa especial, fazendo com que a fração defeituosa do processo sofra uma mudança de magnitude $\delta$, isto é, mude de $p_{0}$ para $p_{1}=\delta p_{0} . \mathrm{O}$ processo permanece em $p_{1}$ até que a causa especial seja removida;

3. O processo é monitorado retirando-se do processo amostras de tamanho $n$ a cada $h$ horas;

4. A velocidade de produção é suficientemente alta para justificar a formação de subgrupos racionais.

Além disso, os modelos econômicos são geralmente formulados com base no custo total do procedimento de controle por unidade de tempo. Três fatores de custos são normalmente considerados: o custo de diagnóstico, o custo de reparar o processo e o custo de produzir itens não conformes. Como o processo retorna ao seu estado inicial após o ajuste, o teorema da renovação garante que, o custo médio assintótico do procedimento por unidade de tempo é dado por

$$
L=\frac{E(C)}{E(T)}
$$

em que $C$ e $T$ são as variáveis aleatórias que representam respectivamente o custo do procedimento de controle em um ciclo e a duração de um ciclo. Um ciclo é o intervalo de tempo entre sucessivos ajustes do processo. 
Exemplo 1.2: Considere um processo de produção em que a distribuição do tempo até a mudança do processo é exponencial, com média $1 / \lambda$. Se o número de itens não conformes supera $d$ (denominado valor aceitável), o processo é interrompido por $t_{0}$ horas a um custo médio de $A_{0}$, se a causa especial não for encontrada; e por $t_{1}$ horas a um custo médio de $A_{l}$, caso contrário. Seja $V_{0}$, o valor ganho por unidade de tempo quando o processo opera no estado sob controle; $\mathrm{V}_{1}$, o valor ganho por unidade de tempo quando o processo opera no estado fora de controle; $\alpha$, a probabilidade do número de itens não conformes em uma amostra selecionada superar $d$, quando o processo está no estado de controle; e $P$, a probabilidade do número de itens não conformes superar $d$, em uma amostra particular, quando o processo não está no estado de controle. Assim

$$
\begin{gathered}
\alpha=1-\sum_{x=0}^{d}\left(\begin{array}{l}
n \\
x
\end{array}\right) p_{0}^{x}\left(1-p_{0}\right)^{n-x} \mathrm{e} \\
P=1-\sum_{x=0}^{d}\left(\begin{array}{l}
n \\
x
\end{array}\right) p_{1}^{x}\left(1-p_{1}\right) \cdot{ }^{n-x}
\end{gathered}
$$

Suponha que em um ciclo, a mudança do processo ocorre entre as amostras $m$ e $(m+1)$, com um tempo médio de ocorrência de

$$
\begin{aligned}
\tau & =\int_{m h}^{(m+1) h} \exp \{-\lambda y\} \lambda(y-m h) d y / \int_{m h}^{(m+1) h} \exp \{-\lambda y\} \lambda d y \\
& =\frac{(1-(1+\lambda h) \exp \{-\lambda h)\}}{\lambda(1-\exp \{-\lambda h\}} .
\end{aligned}
$$

Desta maneira, o ganho médio por unidade de tempo é dado por

$$
I=\frac{E(G)}{E(L)}
$$

em que $E(G)$ é o ganho médio de um ciclo e $E(L)$ é a duração média de um ciclo. Têm-se que $I=V_{0}-F$, em que $F$ é o custo médio do procedimento de controle por item produzido. 
De acordo com Duncan (1956), o comprimento de um ciclo de produção consiste em quatro partes: o período em que o processo permanece em controle, dado por $1 / \lambda$; o período em que o processo sai do estado de controle, dado por $h / P-\tau$; o período destinado à procura de causas especiais associadas a alarmes falsos, dado por $\alpha t_{0}(1 / \lambda-\tau) / h$; e o período destinado à procura de causas especiais associadas alarmes verdadeiros, $t_{1}$. Assim,

$$
E(L)=1 / \lambda+h / P-\tau+\alpha t_{0}(1 / \lambda-\tau) / h+t_{1}
$$

e o ganho médio em um ciclo é expresso por

$$
E(G)=V_{0} / \lambda+V_{1}(h / P-\tau)-\alpha A_{0}(1 / \lambda-\tau) / h-A_{1}-(b+c n)(1 / \lambda+h / P-\tau) / h
$$

Após algumas simplificações, obtêm-se o custo médio esperado do procedimento de controle, por unidade de tempo

$$
F=\frac{\lambda M B_{1}+T B_{0}+\lambda W+(b+c n)\left(1+\lambda B_{1}\right) / h}{1+\lambda B_{1}+t_{0} B_{0}+\lambda t_{1}},
$$

em que $B_{0}=\alpha(1-\lambda \tau) / h, \quad B_{1}=h / P-\tau, \quad M=V_{0}-V_{1}, \quad T=A_{0}+V_{0} t_{0} \quad$ e $\quad W=A_{1}+V_{0} t_{1}$. Assim, deve-se, por pesquisa direta, determinar os valores $h, n$ e $d$ que minimizam $F$.

Uma outra classe de procedimentos para monitorar processos por atributos são os procedimentos introduzidos por Taguchi $(1981,1984,1985)$ e Taguchi et. al. (1989). Taguchi e seu colaboradores tratam o planejamento desses procedimentos por critérios econômicos. Eles envolvem apenas a inspeção de um item retirado da linha de produção, a cada intervalo fixo de $m$ itens produzidos. Se o item inspecionado é diagnosticado como não conforme, o processo é interrompido para ajuste de modo que ao ser reiniciado, as características de interesse dos itens produzidos atendam novamente às especificações. Uma correção da expressão analítica parra o custo médio do procedimento de controle, por item produzido, utilizado por Taguchi é o objeto de estudo dessa dissertação. Este será apresentado e discutido em detalhes no Capítulo 2.

No capítulo 3, será apresentada uma correção da expressão analítica para o custo médio assintótico do procedimento de controle, por item produzido, utilizada por Taguchi 
$(1981,1984,1985)$ e Taguchi et. al. (1989). Esta correção foi introduzida por Nayebpour e Woodall (1993) utilizando uma modelagem alternativa para o mecanismo de falha do processo e será desenvolvida de forma unificada neste Capítulo, a partir de um novo modelo explicitamente caracterizado. Abordaremos também a questão da estimação dos parâmetros do processo a partir de dados observados no procedimento de controle, com particular ênfase no ponto de mudança ou instante da falha do processo. 


\section{Capítulo 2}

\section{O Método de Controle de Taguchi para Atributos com Diagnóstico Perfeito}

Considere um processo de produção onde inspeções periódicas nos itens produzidos são realizadas para assegurar que as características de qualidade do produto satisfaçam as especificações exigidas. Os itens inspecionados são classificados como conformes, se atenderem a essas especificações, ou não conformes, caso contrário.

A inspeção é feita retirando-se um item da linha de produção a cada intervalo fixo de $m$ itens produzidos. O item retirado é analisado e diagnosticado como conforme ou não conforme. Se o item é diagnosticado como não conforme, para-se o processo para ajuste ou reparo de modo que, ao ser reiniciado, as características de interesse dos itens atendam novamente as especificações. Supõe-se que não há erro de diagnóstico, isto é, as probabilidades de diagnosticar um item como não conforme quando ele na verdade atende as especificações e de diagnosticar um item como conforme quando ele na verdade não atende as especificações são nulas. Supõe-se também que a partir do momento em que um item não conforme é inspecionado até o momento em que o processo é interrompido para ajuste pode haver um "atraso" em que são produzidos $l$ itens. Durante este período as inspeções são suspensas.

Diz-se que o processo está sob controle quando produz apenas itens conformes. Quando uma causa especial faz com que o processo passe a produzir itens não conformes, dizse que o processo mudou para o estado fora de controle. O retorno ao estado de controle requer intervenção no processo para repará-lo. Quando o processo retorna ao estado de 
controle, inicia-se um novo ciclo de produção. Os intervalos entre ajustes sucessivos são denominados ciclos. Os itens não conformes produzidos em um ciclo podem ser localizados por inspeção retrospectiva. A inspeção desses itens evita que os mesmos sejam enviados para processos posteriores ou para o mercado.

Taguchi desenvolveu um modelo para determinar o intervalo de diagnóstico $(m)$ que minimiza o custo do procedimento de controle por item produzido, levando-se em conta o custo de inspeção, o custo da produção de itens não conformes, o custo de reparar o processo e de interromper o processo durante o período de reparo. Neste desenvolvimento foram considerados dois casos: no primeiro caso, que será apresentado na Seção 2.1, supõe que ao mudar para o estado fora de controle, o processo passa a produzir somente itens não conformes; no segundo caso, que será apresentado na Seção 2.2, supõe que ao mudar para o estado fora de controle, $\pi \times 100 \%$ dos itens produzidos são não conformes $(0<\pi<1)$. Embora os resultados produzidos por Taguchi e seus colaboradores sejam relativamente simples, as suposições e aproximações utilizadas para obtê-los não são fundamentadas em um modelo estatisticamente bem definido. Uma discussão a respeito dos problemas e limitações da abordagem de Taguchi e seus colaboradores será apresentada na Seção 2.3.

\subsection{Abordagem de Taguchi para o Caso I}

Com o objetivo de determinar o intervalo ótimo de diagnóstico os seguintes fatores de custo são considerados:

(2.1) $C_{d}$ : o custo de um item não conforme produzido;

(2.2) $C_{i}$ : o custo de amostragem e inspeção de um item produzido;

(2.3) $C_{a}$ : o custo de ajuste do processo de produção, incluindo o custo de reparo e o custo de parar o processo de produção para repará-lo.

Observação: Taguchi e seus colaboradores sugerem expressar $C_{a}$ por:

$$
C_{a}=C_{1} t+C_{2},
$$


em que $C_{l}$ é o custo de interromper o processo por unidade de tempo; $t$ é o tempo médio que o processo permanece interrompido para ajuste e $C_{2}$ é o custo de reparo, incluindo mão-de-obra, material e equipamento.

Uma visão gráfica do processo com os fatores de custo considerados acima é apresentada na Figura 2.1.

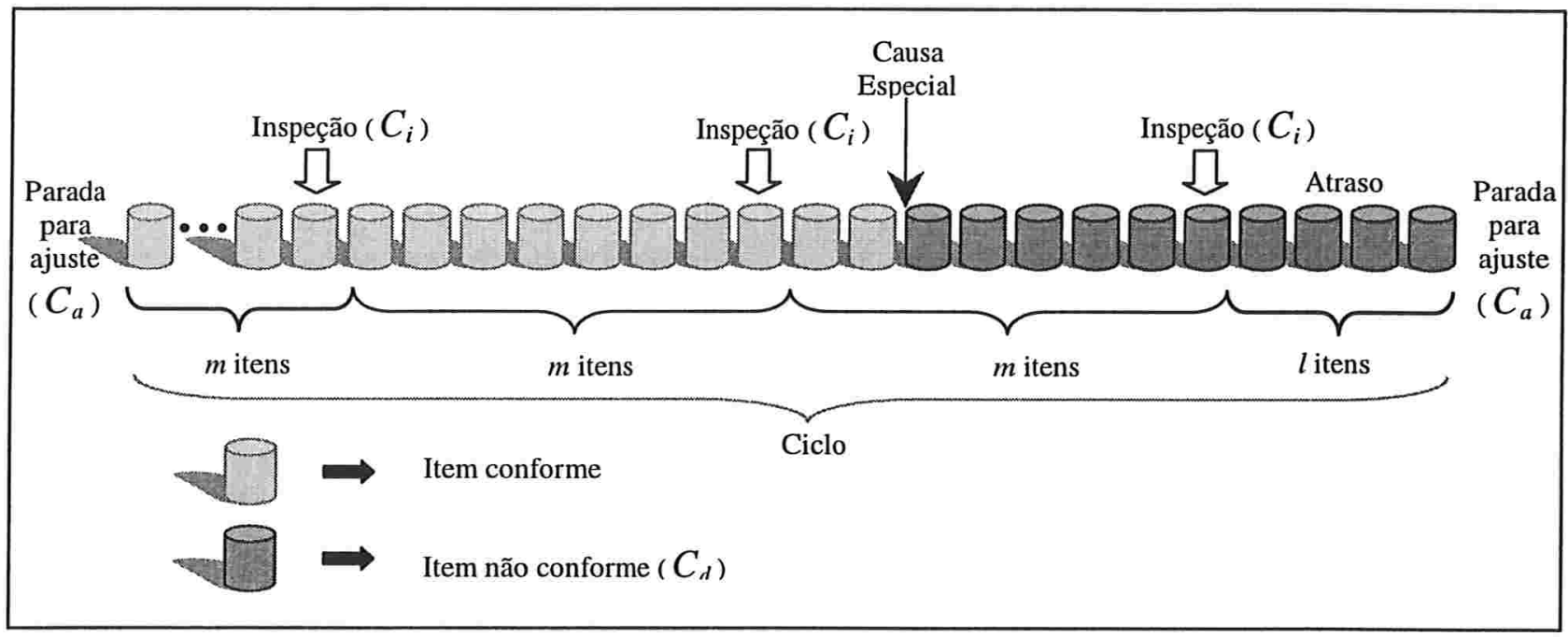

Figura 2.1: Realização típica de um ciclo em que a fração defeituosa do processo muda de 0 para 1.

A função objetivo considerada é o custo médio do procedimento de controle, por item produzido, expressa por

$$
L=\frac{C_{i}}{m}+\frac{m+1}{2} \times \frac{C_{d}}{\bar{u}}+\frac{C_{a}}{\bar{u}}+l \times \frac{C_{d}}{\bar{u}} .
$$

em que $\bar{u}$ é o número médio de itens produzidos em um ciclo.

Segundo Taguchi, o primeiro termo da expressão (2.1) refere-se ao custo esperado de inspeção em um ciclo, por item produzido. A justificativa para a este termo, é que a cada $m$ itens produzidos há um acréscimo $C_{i}$ no custo do procedimento de controle. O segundo termo, refere-se ao custo esperado de itens não conformes em um ciclo por item produzido. Taguchi e seus colaboradores argumentam que como a mudança de estado no processo ocorre no último intervalo de diagnóstico, o número de itens não conformes produzidos em um ciclo pode variar entre 1 e $m$, de tal forma que são produzidos em média, $\frac{1}{m}(1+\ldots+m)=\frac{m+1}{2}$ itens não conformes. Deve ser suposto, evidentemente, que estes resultados possíveis são igualmente 
prováveis. O terceiro termo da expressão (2.1), refere-se ao custo esperado de ajuste em um ciclo, por item produzido. A justificativa para este termo, é que a cada ciclo há um acréscimo $C_{a}$ no custo do procedimento. E o quarto termo, ao custo esperado do atraso no ajuste em um ciclo, por item produzido Taguchi argumenta que há um acréscimo $l C_{d}$ no custo do procedimento a cada ciclo do processo.

Assim, o primeiro intervalo de diagnóstico que minimiza a expressão (2.1) é obtido resolvendo-se a equação

$$
\frac{\partial L}{\partial m}=-\frac{C_{i}}{m^{2}}+\frac{C_{d}}{2 \bar{u}}=0
$$

em relação a $m$, cuja solução é

$$
m^{*}=\sqrt{2 \bar{u} \times \frac{C_{i}}{C_{d}}} .
$$

Como (2.2) não leva em consideração o número de itens produzidos durante o período de "atraso" do ajuste, $l$, e o custo do ajuste, $C_{a}$, Taguchi e seus colaboradores sugerem que a expressão (2.2) seja utilizada apenas se as condições

$$
\bar{u} \gg l \text { e } C_{d} \gg \frac{C_{a}}{\bar{u}}
$$

forem satisfeitas. O Exemplo 2.1 apresentado a seguir ilustra o impacto destas condições na perda mínima esperada.

Exemplo 2.1: Berry (1974) apresenta resultados do monitoramento da produção de tornos. Um sistema de monitoramento computadorizado foi instalado para minimizar a perda total dos 160 tornos automáticos de uma planta. Os parâmetros do processo de produção são:

$$
C_{d}=\$ 5,00, C_{i}=\$ 150,00, l=1.000 \text { itens }, \bar{u}=4.000 \text { itens e } C_{a}=\$ 4.000 \text {. }
$$

Pela equação (2.2), $m^{*}=\sqrt{\frac{2 \times 4000 \times 150}{5}}=490$ itens e a perda média mínima, calculada pela equação (2.1) é de $\$ 2,86$. 
Supondo agora que:

$$
C_{d}=\$ 5,00, C_{i}=\$ 150,00, l=10 \text { itens }, \bar{u}=4.000 \text { itens e } C_{a}=\$ 40,
$$

teremos $m^{*}=490$ itens e perda média mínima de $\$ 0,32985$.

$\mathrm{Na}$ situação em que as condições (2.2.a) não são satisfeitas, Taguchi e seus colaboradores sugerem substituir $\bar{u}$ por $\bar{u}+m / 2 \mathrm{em}(2.1)$, argumentando que quando o $k$ ésimo item inspecionado é não conforme, a mudança de estado do processo ocorre entre os diagnósticos $k$-1 e $k$, produzindo, em média, $\frac{m}{2}$ itens não conformes. Assim, o número esperado de itens produzidos em um ciclo seria $\bar{u}+m / 2$ ao invés de $\bar{u}$. Com esta substituição e aproximando

$$
\frac{1}{\bar{u}+m / 2}=\frac{1}{\bar{u}} \times \frac{1}{1+m / 2 \bar{u}}=\frac{1}{\bar{u}}\left(1-\frac{m}{2 \bar{u}+m}\right) \text { por } \frac{1}{\bar{u}}\left(1-\frac{m}{2 \bar{u}}\right)
$$

obtêm-se

$$
L=\frac{C_{i}}{m}+\frac{1}{\bar{u}}\left(1-\frac{m}{2 \bar{u}}\right)\left(\frac{m+1}{2} C_{d}+C_{a}+l C_{d}\right)
$$

A expressão para o intervalo ótimo de diagnóstico é obtida resolvendo-se a equação

$$
\frac{\partial L}{\partial m}=-\frac{C_{i}}{m^{2}}+\frac{C_{d}}{2 \bar{u}}+\frac{(2 m+1) C_{d}}{4 \bar{u}}-\frac{1}{2 \bar{u}^{2}}\left(C_{a}+l C_{d}\right)=0 .
$$

Observação: a expressão dada em (2.4) é a versão corrigida apresentada por Nayebpour (1991 p.15) da equação dada por Taguchi (1989 p.107).

Na solução de (2.4), Taguchi e seus colaboradores omitem o terceiro termo da derivada, argumentando ser ele bem menor que o segundo. Com essa simplificação obtêm-se 


$$
m=\sqrt{\frac{2 \bar{u} C_{i}}{C_{d}-\frac{C_{a}}{\bar{u}}-\frac{l C_{d}}{\bar{u}}}} .
$$

Utilizando a aproximação

$$
\begin{aligned}
& \left(C_{d}-\frac{C_{a}}{\bar{u}}-\frac{l C_{d}}{\bar{u}}\right)^{-1}=\left(\left(C_{d}-\frac{C_{a}}{\bar{u}}\right)\left(1-\frac{l C_{d} / \bar{u}}{C_{d}-\left(C_{a} / \bar{u}\right)}\right)\right)^{-1}= \\
& =\left(C_{d}-\frac{C_{a}}{\bar{u}}\right)^{-1}\left(\frac{C_{d}-C_{a} / \bar{u}+\left(-l C_{d} / \bar{u}+l C_{d} / \bar{u}\right)}{C_{d}-C_{a} / \bar{u}-l C_{d} / \bar{u}}\right)= \\
& =\left(C_{d}-\frac{C_{a}}{\bar{u}}\right)^{-1}\left(1+\frac{l C_{d} / \bar{u}}{C_{d}-C_{a} / \bar{u}-l C_{d} / \bar{u}}\right) \approx \\
& \approx\left(C_{d}-\frac{C_{a}}{\bar{u}}\right)^{-1}\left(1+\frac{l C_{d} / \bar{u}}{C_{d}}\right)=\left(C_{d}-\frac{C_{a}}{\bar{u}}\right)^{-1}\left(1+\frac{l}{\bar{u}}\right)
\end{aligned}
$$

obtém-se uma expressão alternativa para o intervalo ótimo de diagnóstico que é dada por

$$
m^{* *}=\sqrt{2 \times \frac{(\bar{u}+l) C_{i}}{C_{d}-\frac{C_{a}}{\bar{u}}}}
$$

O Exemplo 2.2 apresentado a seguir foi usado para ilustrar a diferença entre os resultados obtidos pelas equações (2.2) e (2.6) no cálculo do intervalo ótimo de diagnóstico.

Exemplo 2.2: Considere a situação apresentada no Exemplo 2.1. Os parâmetros do processo de produção são os seguintes:

$$
C_{d}=\$ 5,00, C_{i}=\$ 150,00, l=1000 \text { itens }, \bar{u}=4.000 \text { itens e } C_{a}=\$ 4.000 \text {. }
$$

Pela equação (2.6), $m^{* *}=\sqrt{2 \times \frac{(\bar{u}+l) C_{i}}{C_{d}-\frac{C_{a}}{\bar{u}}}}=\sqrt{\frac{2 \times 5.000 \times 150}{5-4.000 / 4.000}}=612$ itens e a perda média mínima, calculada pela equação (2.1) é de $\$ 2,88$. 
Observe que, a equação (2.2) resulta em inspecionar um item a cada 490 itens produzidos e a perda média mínima, calculada pela equação (2.1) é de $\$ 2,86$.

Supondo novamente que:

$$
C_{d}=\$ 5,00, C_{i}=\$ 150,00, l=10 \text { itens }, \bar{u}=4.000 \text { itens e } C_{a}=\$ 40,
$$

Pela equação (2.6), $m^{* *}=549$ itens e a perda média mínima, calculada pela equação (2.1) é de $\$ 1,63$.

Observe que, a equação (2.2) resulta em inspecionar um item a cada 490 itens produzidos e a perda média mínima, calculada pela equação (2.1) é de \$0,329.

A comparação entre as expressões do intervalo ótimo de diagnóstico está apresentada na Tabela 2.1

\begin{tabular}{c|cc|cc}
\hline \hline \multirow{2}{*}{$\begin{array}{c}\text { Número de itens } \\
\text { produzidos no }\end{array}$} & \multicolumn{4}{|c}{ Intervalo Ótimo de Diagnóstico } \\
\cline { 2 - 5 } período de atraso $(l)$ & \multicolumn{2}{|c|}{ Equação (2.2) } & \multicolumn{2}{c}{ Equação (2.6) } \\
\cline { 2 - 5 } & $m^{*}$ & $L$ & 612 & 2,88 \\
\hline$l=1.000$ & 490 & 2,86 & 548 & 1,63 \\
\hline$l=10$ & 490 & 0,33 & $m^{* *}$ \\
\hline \hline
\end{tabular}

Tabela 2.1 : Cálculo dos intervalos de diagnóstico e perda média mínima considerando $C_{d}=$ $\$ 5,00, C_{i}=\$ 150,00, \bar{u}=4.000$ itens e $C_{a}=\$ 4.000$.

Taguchi afirma que, embora a diferença no custo pareça ser insignificante, existe uma grande diferença entre os intervalos de inspeção obtidos pelas expressões (2.2) e (2.6). A diferença entre os resultados diminui quando as condições dadas em (2.2.a) são satisfeitas. Caso isso não aconteça, é recomendado usar a expressão (2.6). 


\subsection{Abordagem de Taguchi para o Caso 2}

Uma visão gráfica do processo com os fatores de custo é apresentado na Figura 2.2.

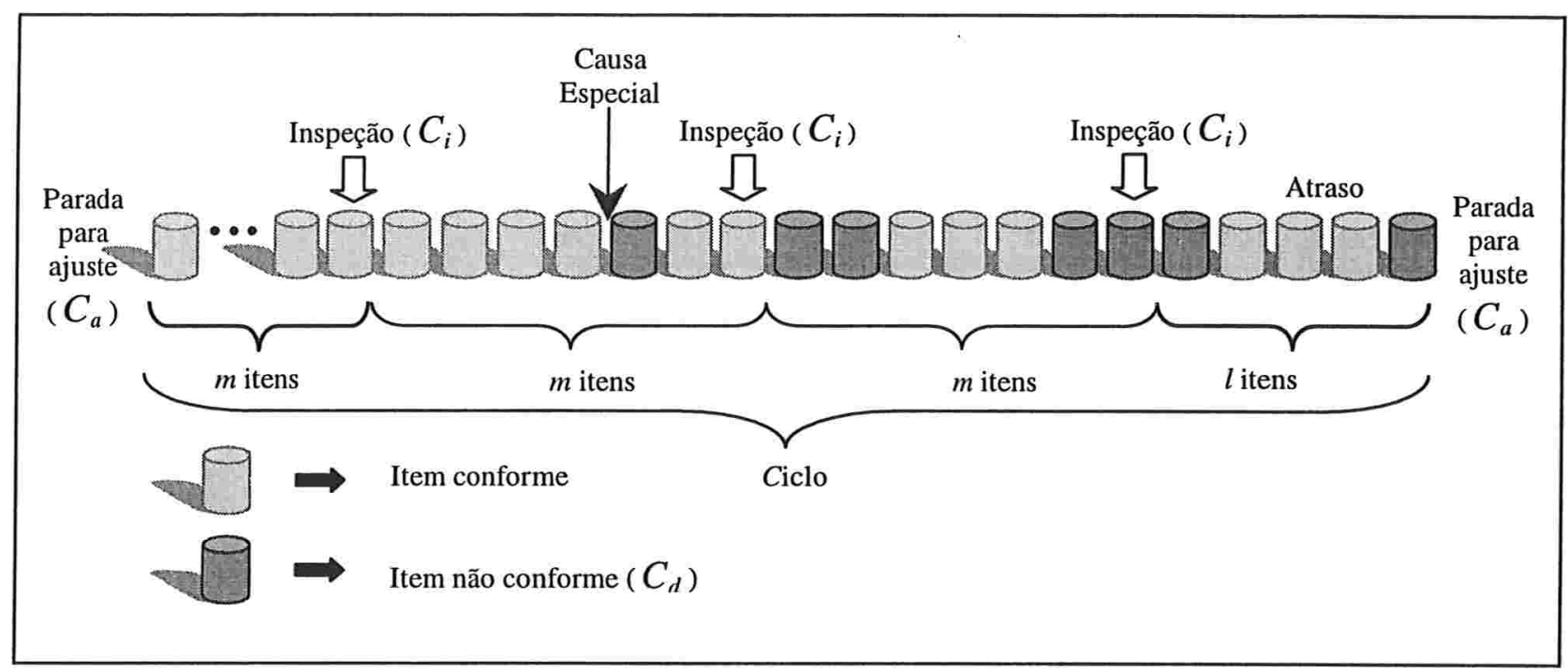

Figura 2.2 - Realização típica de um ciclo em que a fração defeituosa do processo outros muda de 0 para $\pi(0<\pi<1)$.

Para determinar o intervalo de diagnóstico ótimo no Caso II, é considerado $C_{D}$, o custo esperado de um item não conforme que não pode ser identificado no estágio de produção e é enviado para processos posteriores ou para o mercado e os fatores de custo $C_{d}, C_{i}$ e $C_{a}$ como definidos na Seção 2.1. Na prática $C_{D} \gg C_{d}$, uma vez que $C_{D}$ é constituído por fatores de custo imponderáveis, como o custo da insatisfação do cliente ao receber um item não conforme.

Taguchi e seus colaboradores expressam a custo esperado de itens não conformes produzidos em um ciclo como

$$
C_{d+D}=k_{1} C_{d}+k_{2} C_{D}
$$

em que $C_{d+D}$ é o custo esperado de um item não conforme em um ciclo, $\pi$ a fração de itens não conformes produzidos após a mudança do processo, $k_{1}$ e $k_{2}$ dados pelas seguintes expressões 


$$
\begin{aligned}
& k_{1}=\left(\pi \times \frac{(m+1) \pi}{2}\right)+(\pi(1-\pi) \times m \pi)^{+}\left(\pi(1-\pi)^{2} \times m \pi\right)+\left(\pi(1-\pi)^{3} \times m \pi\right)+\ldots= \\
& =\frac{(m+1) \pi^{2}}{2}+m \pi\left[(1-\pi) \pi+(1-\pi)^{2} \pi+\ldots\right]=\frac{(m+1) \pi^{2}}{2} \times m \pi(1-\pi) \pi
\end{aligned}
$$

$\mathrm{e}$

$$
\begin{aligned}
& k_{2}=(\pi \times 0)+\left(\pi(1-\pi) \times \frac{(m+1) \pi}{2}\right)+\pi(1-\pi)^{2}\left(\frac{(m+1) \pi}{2}+m \pi\right)+\pi(1-\pi)^{3}\left(\frac{(m+1) \pi}{2}+2 m \pi\right)+\ldots= \\
& =\frac{(m+1) \pi}{2}\left[(1-\pi) \pi+(1-\pi)^{2} \pi+\ldots\right]+m \pi\left[(1-\pi)^{2} \pi+2(1-\pi)^{3} \pi+\ldots+(i-2)(1-\pi)^{i-1} \pi+\cdots\right]= \\
& =\frac{(m+1)}{2} \times(1-\pi) \pi+m(1-\pi)^{2}
\end{aligned}
$$

Assim,

$$
C_{d+D}=\left\{\frac{(m+1) \pi^{2}}{2}+m \pi(1-\pi)\right\} C_{d}+\left\{\frac{(m+1)}{2} \times(1-\pi) \pi+m(1-\pi)^{2}\right\} C_{D}
$$

Taguchi e seus colaboradores justificam esta expressão construindo a Tabela 2.2 (Taguchi et al., 1989, p. 110). Supõe-se que os itens não conformes produzidos entre o diagnóstico que detectou anomalia no processo e o anterior, podem ser identificados por inspeção retrospectiva e removidos; os demais não podem ser identificados e são enviados para processos posteriores ou para o mercado. Para obter a Tabela 2.1, supõe-se que o número de itens não conformes produzidos no intervalo de diagnóstico onde ocorreu a mudança no processo pode variar entre 1 e $m$, com a mesma probabilidade, de tal forma que são produzidos em média $\frac{(m+1)}{2} \pi$ itens não conformes neste intervalo, desde que a fração de itens não conformes produzidos após a mudança no processo seja $\pi$. 


\begin{tabular}{cccc}
\hline $\begin{array}{c}\text { Ponto } \\
\text { de }\end{array}$ & $\begin{array}{c}\text { Probabilidade de } \\
\text { detectar uma }\end{array}$ & $\begin{array}{c}\text { Número esperado de } \\
\text { itens não conformes que }\end{array}$ & $\begin{array}{c}\text { Número esperado de itens } \\
\text { não conformes não } \\
\text { identificados }\end{array}$ \\
\hline 1 & $\pi$ & $\begin{array}{c}\text { anomalia no processo } \\
\text { podem ser identificados }\end{array}$ & 0 \\
2 & $(1-\pi) \pi$ & $m \pi$ & 0 \\
3 & $(1-\pi)^{2} \pi$ & $m \pi / 2$ & $(m+1) \pi / 2$ \\
4 & $(1-\pi)^{3} \pi$ & $m \pi$ & $(m+1) \pi / 2+2 m \pi$ \\
$\vdots$ & $\vdots$ & $\vdots$ & $\vdots$ \\
$i$ & $(1-\pi)^{\mathrm{i}-1} \pi$ & $m \pi$ & $(m+1) \pi / 2+(i-2) m \pi$ \\
$\vdots$ & $\vdots$ & $\vdots$ & $\vdots$ \\
\hline
\end{tabular}

Tabela 2.2 - Número esperado de itens não conformes em diferentes pontos de inspeção.

Se $\pi \approx 1$, a equação (2.7) reduz a equação (2.2) do caso I, e segundo Taguchi, deve ser usada para determinar o intervalo ótimo de diagnóstico. Entretanto, se $\pi \approx 0$ a equação (2.7) se reduz a $m C_{D}$. Taguchi argumenta que $m C_{D}$ corresponde a perda $\frac{(m+1)}{2} C_{d}$ da equação (2.1). Considerando $\frac{(m+1)}{2} \approx \frac{m}{2}$ e substituindo $2 C_{D}$ por $C_{d}$ na equação (2.1), obtém-se o custo médio do procedimento de controle, por item produzido, para o Caso II, que é expresso por

$$
L=\frac{C_{i}}{m}+(m+1) \frac{C_{D}}{\bar{u}}+\frac{C_{a}}{\bar{u}}+l \times \frac{C_{d}}{\bar{u}} .
$$

Os outros termos da expressão são justificados por Taguchi e seus colaboradores usando os mesmos argumentos da Seção 2.1.

O intervalo de diagnóstico que minimiza o custo do procedimento de controle por item produzido é expresso por

$$
m^{* * *}=\sqrt{2 \times \frac{(\bar{u}+l) C_{i}}{2 C_{D}-\frac{C_{a}}{\bar{u}}}}
$$

que é determinado igualando a zero a derivada da equação (2.8) e resolvendo em relação a $m$. 
Taguchi afirma que se $\pi<1$ é possível identificar todos itens não conformes voltando ao ponto onde o processo mudou para o estado fora de controle. Assim, todos os itens não conforme podem ser identificados e o custo $C_{D}$ se equivale ao custo $C_{d}$. Neste caso, a equação (2.8) reduz-se a

$$
L=\frac{C_{i}}{m}+(m+1) \frac{C_{d}}{\bar{u}}+\frac{C_{a}}{\bar{u}}+l \times \frac{C_{d}}{\bar{u}}
$$

e o intervalo ótimo de diagnóstico é expresso por

$$
m^{* * *}=\sqrt{2 \times \frac{(\bar{u}+l) C_{i}}{2 C_{d}-\frac{C_{a}}{\bar{u}}}},
$$

Taguchi afirma ainda que, a equação (2.11) resulta numa superinspeção do processo de produção quando $\pi$ não é próximo de zero e recomenda usar as expressões (2.2) e (2.6) quando $\pi \geq 0.5$.

O Exemplo apresentado a seguir ilustra como a escolha do intervalo de diagnóstico atua no custo esperado em um ciclo por item produzido (Taguchi et al., 1989).

Exemplo 2.3: Num processo de produção, placas de circuito impresso são montadas com dispositivos soldados. As ligações soldadas de cada placa são inspecionadas para detectar falta de dispositivos, ligações indevidas e ausência de filetes de solda. Esta inspeção é realizada em diferentes pontos ao longo da linha de produção. A perda causada pela produção de uma placa de circuito não conforme é $\$ 3,00$ e o custo da inspeção por placa é $\$ 10,00$. $O$ processo é ajustado em um intervalo médio de 500 placas, a um custo de $\$ 25,00$. O atraso do ajuste no processo, expresso em itens de produção é de 5 placas. Quando um item não conforme é diagnosticado os outros itens não conformes produzidos no período são retirados do processo. Os parâmetros do processo de produção são os seguintes:

$$
C_{d}=\$ 30,00, C_{i}=\$ 10,00, l=5 \text { itens }, \bar{u}=500 \text { itens e } C_{a}=\$ 25 \text {. }
$$


Pela equação (2.6), $m^{* *}=\sqrt{2 \times \frac{(500+5) 10}{30-\frac{25}{500}}} \approx 18$ itens e a perda média mínima, calculada pela equação (2.1) é de $\$ 1,48$.

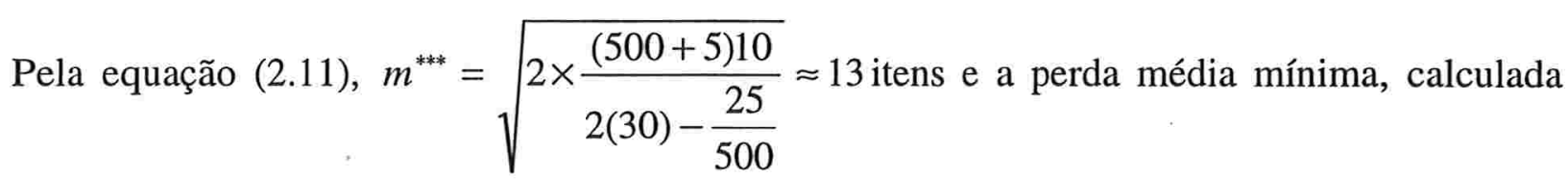
pela equação (2.10) é de $\$ 1,96$.

\subsection{Comentários sobre a Abordagem de Taguchi}

Para determinar as expressões do custo médio do procedimento de controle dadas em (2.1), (2.3), (2.8) e (2.10) e seus correspondentes intervalos ótimos de diagnóstico, Taguchi não basea-se em um modelo probabilístico. Assim, estabelece algumas aproximações e suposições não fundamentadas e expressões de custo e intervalo ótimo de diagnóstico questionáveis.

Ao formular o custo esperado de inspeção em um ciclo por item produzido, é argumentado que a cada $m$ itens produzidos há um acréscimo de $C_{i}$ no custo do procedimento. Entretanto, se $k$ representa o número médio de inspeções em um ciclo, o custo médio de inspeção em um ciclo, por item produzido devia ser dado por $\frac{k C_{i}}{\bar{u}}$ que difere do termo utilizado por Taguchi e seus colaboradores .

O custo esperado de itens não conformes em um ciclo por item produzido, formulado para o Caso I é baseado no número médio de itens não conformes do último intervalo de diagnóstico. Observe, entretanto que, o "último intervalo de inspeção" pode ser o primeiro, o segundo, etc... de tal forma que este valor é apenas uma esperança condicional. Mais precisamente, se $\theta$ for o número de itens não conformes no ciclo 


$$
\begin{aligned}
& \mathrm{E}(k m-\theta=i / m(k-1) \leq \theta \angle k m)= \\
& \sum_{i=1}^{m} i \times \mathrm{P}(\theta=k m-i / m(k-1) \leq \theta \angle k m)= \\
& \sum_{i=1}^{m} i \times \frac{1}{m}=\left[(1+m) \times \frac{m}{2}\right] \times \frac{1}{m}=\frac{m+1}{2} .
\end{aligned}
$$

Ainda assim, se são produzidos $l$ itens não conformes durante o período de atraso, temos, em média, $\frac{m+1}{2}+l$ itens não conformes em um ciclo. Assim, o custo médio de itens não conformes em um ciclo, por item produzido é

$$
\left(\frac{m+1}{2}+l\right) \times \frac{C_{d}}{\bar{u}}=\frac{m+1}{2} \times \frac{C_{d}}{\bar{u}}+l \times \frac{C_{d}}{\bar{u}},
$$

que na expressão (2.1) é o segundo e o último do termo. Taguchi se refere ao último termo como o custo esperado do atraso em um ciclo, por item produzido.

Os intervalo de diagnóstico que minimizam o custo do procedimento de controle por item produzido, dados em (2.2), (2.6), (2.9) e (2.11) são obtidos pela derivada parcial das expressões (2.1), (2.3) e (2.8) e (2.10), respectivamente. Observe que, qualquer dependência entre $\bar{u}$ e $m$ é ignorada na derivação.

$\mathrm{Na}$ determinação da expressão do custo médio do procedimento de controle dada em (2.3) e de $m^{* *}$ expresso em (2.6), o número de itens produzidos em um ciclo é definido como $\bar{u}+m / 2$, ao invés $\bar{u}$. Note que, neste ponto, a definição inicial de $\bar{u}$ é alterada. Para obter (2.6), Taguchi simplifica (2.5) usando uma aproximação que só é válida se $\bar{u} \gg>$ e $C_{d}>>$ $C_{a} / \bar{u}$. Entretanto, o uso da equação (2.6) é recomendado justamente quando estas suposições não são satisfeitas. Observe que, ambas as equações (2.5) e (2.6) apresentam a mesma complexidade e (2.6) não minimiza (2.3) ou (2.1). No Exemplo 2.2, a equação (2.6) é usada para determinar o intervalo de diagnóstico, entretanto o custo esperado em um ciclo por item produzido não é determinado pela equação (2.3).

No Caso II, para a determinação expressão do custo médio do procedimento de controle dada em (2.8) e o correspondente intervalo de diagnóstico em (2.9), Taguchi considera $\pi \approx 0$. Assim, estas equações são válidas neste caso. Observe que, o último termo da expressão (2.8), deveria ser expresso por $l \pi \times \frac{C_{d}}{\bar{u}}$. A expressão sugere que todos os itens 
produzidos durante o período de atraso no ajuste do processo são não conformes, ou seja, $\pi=$ 1. Note também que, as equações (2.9) e (2.11) não dependem do valor de $\pi$.

Taguchi afirma que se $\pi<1$ é possível detectar todos os itens não conformes voltando ao ponto onde o processo muda para o estado fora de controle. Entretanto, uma vez que não é determinado o ponto de mudança do processo, a inspeção retrospectiva torna-se inviável; caso fosse, não foi incluído o custo da inspeção retrospectiva na função de perda esperada (2.8).

Pode-se concluir que, tanto para o Caso I quanto para o caso II, Taguchi estabelece algumas aproximações e suposições que são questionáveis, uma vez que não são baseadas em um modelo estatisticamente bem definido. Consequentemente, as expressões do custo médio do procedimento de controle e dos intervalos ótimos de diagnóstico possuem problemas substanciais em sua determinação, e as recomendações indicadas foram feitas sem nenhum embasamento matemático. 


\section{Capítulo 3}

\section{A Correção de Nayebpour and Woodall}

Neste capítulo será apresentada uma correção da expressão analítica para o custo médio assintótico do procedimento de controle, por item produzido, utilizada por Taguchi $(1981,1984,1985)$ e Taguchi et. al. (1989). Esta correção foi introduzida por Nayebpour and Woodall (1993), utilizando uma modelagem alternativa para o mecanismo de falha do processo. Entretanto, um pequeno erro em duas expressões desenvolvidas por Nayebpour and Woodall (1993) foi apontado por Borges et al. (2000) ao estenderem os resultados de Nayebpour and Woodall (1993) para a situação em que as inspeções são imperfeitas. Segundo Borges et al. (2000) uma consideração equivocada no modelo utilizado levou ao erro. Por esse motivo, a correção será desenvolvida, de forma unificada, neste Capítulo, a partir de um novo modelo. Abordaremos também a questão da estimação dos parâmetros do processo a partir de dados observados no procedimento de controle, com particular ênfase no ponto de mudança ou instante da falha do processo. Como veremos, esta questão inferencial está inteiramente relacionada com a recomendação técnica de inspecionar retrospectivamente, em cada ciclo, apenas os itens produzidos durante o último intervalo de amostragem e o atraso.

Como o processo retorna ao seu estado inicial após o ajuste, o teorema da renovação nos garante que, o custo médio assintótico do procedimento por item produzido é dado por

$$
L=\frac{E(C)}{E(T)}
$$


em que $C$ e $T$ são as variáveis aleatórias que representam o custo do procedimento de controle em um ciclo e o número de itens produzidos em um ciclo. O intervalo ótimo de diagnóstico é dado pelo valor de $m$ que minimiza $L$.

Para obtermos a expressão analítica de $L$ em função dé $m$, utilizaremos um processo $\left\{\left(X_{i}, \Theta_{i}\right): i \geq 0\right\}$, em que as variáveis aleatórias binárias, $X_{i}, i \geq 1$, representarão a condição do $i$-ésimo item produzido. Mas especificamente,

$$
\begin{aligned}
X_{i} & =0, \text { se o } i \text {-ésimo item produzido é conforme; } \\
& =1 \text {, caso contrário. }
\end{aligned}
$$

As variáveis aleatórias $\Theta_{i}, i \geq 1$, representarão a fração defeituosa do processo do instante da produção do $i$-ésimo item e o mecanismo de falha será descrito pelo comportamento estocástico de $\left\{\Theta_{i}: i \geq 0\right\}$. Especificamente, vamos supor que esta seqüência é uma cadeia de Markov com espaço de estados $E=\{0, \pi\}$, matriz de transição $M=\left[\begin{array}{cc}q & (1-q) \\ 0 & 1\end{array}\right]$, e distribuição inicial $P\left(\theta_{0}=0\right)=1$ concentrada no ponto $0 \in E$. Supõe-se ainda que, dados $\Theta_{1}=\theta_{1}, \ldots, \Theta_{n}=\theta_{n}, X_{0}, X_{1}, \ldots, X_{n}$ são independentes e tais que $P\left\{X_{i}=1 \mid \Theta_{i}=\theta_{i}\right\}=1$ $-P\left\{X_{i}=0 \mid \Theta_{i}=\theta_{i}\right\}=\theta_{i}$. Observe que estamos de fato descrevendo um procesšo que permanece na condição de "controle", $\Theta_{i}=0$, até que uma causa especial produza uma "absorção" na condição "fora de controle", $\Theta_{i}=\pi$. Definimos ainda a variável aleatória $W=\inf \left\{i \geq 1: \Theta_{i}=\pi\right\}-1=\sup \left\{i \geq 1: \Theta_{i}=0\right\}$, que representa o "ponto" de mudança do processo. Note que, $W$ é também o número de itens conformes produzidos em um ciclo. Portanto,

$$
P\{W \geq k\}=\mathrm{P}\left\{\Theta_{1}=0, \ldots, \Theta_{k}=0\right\}=q^{k}, \quad k \geq 0,
$$

isto é, $W$ tem distribuição geométrica com média $q /(1-q)$. O parâmetro $q$ representa, portanto, a probabilidade do processo permanecer sob controle.

O processo de produção é definido considerando as suposições feitas por Taguchi, apresentadas no Capítulo 2. O caso I, em que $\pi=1$, pode ser representado pela Figura 2.1; e o caso II, em que $\pi<1$, pela Figura 2.2 .

Para determininar o número médio de itens produzidos em um ciclo, $E(T)=m E(\xi)+l$, em que $\xi$ denota o número de inspeções, observe que 


$$
\begin{aligned}
E(\xi) & =\sum_{k=0}^{\infty} P\{\xi>k\}=1+\sum_{k=1}^{\infty} P\{\xi>k\} \\
& =1+\sum_{k=1}^{\infty} P\left\{X_{m}=0, \ldots, X_{k m}=0\right\} \\
& =1+\sum_{k=1}^{\infty} E\left[P\left\{X_{m}=0, \ldots, X_{k m}=0 \mid W\right\}\right] .
\end{aligned}
$$

Como para $k \geq 1$

$$
\begin{aligned}
P\left\{X_{m}=0, \ldots, X_{k m}=0 \mid W\right\}=1, & \text { se } k m \leq W \\
=(1-\pi)^{k-j}, & \text { se } j m \leq W<(j+1) m, 0 \leq j<k,
\end{aligned}
$$

Isto é,

$$
P\left\{X_{m}=0, \ldots, X_{k m}=0 \mid W\right\}=(1-\pi)^{\left(K-\left[\frac{W}{m}\right] \vee v 0\right.}, k \geq 1,
$$

em que $[x]$ denota o maior inteiro menor ou igual a $x$. Assim

$$
\begin{aligned}
E\left[P \left\{X_{m}=\right.\right. & \left.\left., \ldots, X_{k m}=0 \mid W\right\}\right] \\
& =E\left[(1-\pi)^{\left(k-\left[\frac{W}{m}\right]{ }^{\vee} 0\right.}\right] \\
& =\sum_{j=0}^{\infty} \sum_{w=j m}^{(j+1) m-1}(1-\pi)^{(k-j) \vee 0} q^{w}(1-q) \\
& =\sum_{j=0}^{k-1} \sum_{w=j m}^{(j+1) m-1}(1-\pi)^{k-j} q^{w}(1-q)+\sum_{j=k}^{\infty} \sum_{w=j m}^{(j+1) m-1} q^{w}(1-q) \\
& =\sum_{j=0}^{k-1}(1-\pi)^{k-j} q^{j m}\left(1-q^{m}\right)+\sum_{j=k}^{\infty} q^{j m}\left(1-q^{m}\right) \\
& =(1-\pi)^{k}\left(1-q^{m}\right) \sum_{j=0}^{k-1}\left(\frac{q^{m}}{1-\pi}\right)^{j}+q^{k m} \\
& =(1-\pi)^{k}\left(1-q^{m}\right) \frac{1-\left(\frac{q^{m}}{1-\pi}\right)^{k}}{1-\frac{q^{m}}{1-\pi}}+q^{k m} \\
& =(1-\pi)^{k}\left(1-q^{m}\right) \frac{\left((1-\pi)^{k}-q^{k m}\right) /(1-\pi)^{k}}{\left((1-\pi)-q^{m}\right) /(1-\pi)}+q^{k m} \\
& =(1-\pi)\left(1-q^{m}\right) \frac{(1-\pi)^{k}-q^{k m}}{(1-\pi)-q^{m}}+q^{k m} .
\end{aligned}
$$


Substituindo (3.5) em (3.3) obtêm-se

$$
\begin{aligned}
E(\xi) & =1+\sum_{k=1}^{\infty} E\left[P\left\{X_{m}=0, \ldots, X_{k m}=0 \mid W\right\}\right] \\
& =1+\sum_{k=1}^{\infty}\left[(1-\pi)\left(1-q^{m}\right) \frac{(1-\pi)^{k}-q^{k m}}{(1-\pi)-q^{m}}+q^{k m}\right] \\
& =1+\frac{(1-\pi)\left(1-q^{m}\right)}{\left(1-\pi-q^{m}\right)}\left[\frac{1-\pi}{\pi}-\frac{q^{m}}{\left(1-q^{m}\right)}\right]+\frac{q^{m}}{\left(1-q^{m}\right)} \\
& =\frac{1-\pi}{\pi}+\frac{1}{1-q^{m}} .
\end{aligned}
$$

Logo

$$
E(T)=\frac{m}{1-q^{m}}+\frac{m(1-\pi)}{\pi}+l .
$$

Observe que, para o Caso I, $E(T)$ se reduz a $\frac{m}{1-q^{m}}+l$.

Para determinar o numerador de (3.1), isto é, o custo médio do procedimento de controle em um ciclo, será considerada inicialmente a situação em que não é realizada inspeção retrospectiva no último intervalo de diagnóstico. Assim como em Taguchi $(1981,1984,1985)$ e Taguchi et. al. (1989), são definidos aqui os seguintes fatores de custo: $C_{i}$, o custo de inspeção; $C_{D}$, o custo de um item não conforme produzido e $C_{a}$, o custo de ajuste no processo. Assim,

$$
C=C_{D} \sum_{i=1}^{m \xi+l} X_{i}+C_{i} \xi+C_{a}
$$

Consequentemente,

$$
\begin{aligned}
E(C) & =C_{D} E\left(\sum_{i=1}^{m \xi+l} X_{i}\right)+C_{i} E(\xi)+C_{a} \\
& =C_{D} E\left[E\left(\sum_{i=1}^{m \xi+l} X_{i} \mid \xi, W\right)\right]+C_{i} E(\xi)+C_{a} .
\end{aligned}
$$


Como $P\{W=w, \xi=k\}>0$ somente se $w \geq 0$ e $k m>w$, para $w \geq 0$ e $j \geq 1$, temos

$$
\begin{aligned}
P\left\{W=w, \xi=\left[\frac{w}{m}\right]+j\right\}= & P\{W=w\} P\left\{\xi=\left[\frac{w}{m}\right]+j \mid W=w\right\} \\
& =P\{W=w\} P\left\{X_{m}=0, \ldots, X\left(\left[\frac{w}{m}\right]_{+j-1}\right)_{m}=0, X\left(\left[\frac{w}{m}\right]+j\right)_{m}=1 \mid W=w\right\} \\
& =q^{w}(1-q)(1-\pi)^{j-1} \pi,
\end{aligned}
$$

em que $[x]$ denota o maior inteiro menor ou igual a $x$. Assim

$$
\begin{aligned}
E[E & \left.\left(\sum_{i=1}^{m \xi+l} X_{i} \mid \xi, W\right)\right] \\
& =\sum_{w=0}^{\infty} \sum_{k=1}^{\infty}\left\{\left[\left(\left[\frac{w}{m}\right]+1\right) m-w-1\right] \pi+(k-1)(m-1) \pi+l \pi+1\right\}(1-\pi)^{k-1} \pi q^{w}(1-q) .
\end{aligned}
$$

\section{Como}

$$
\begin{aligned}
\sum_{w=0}^{\infty} \sum_{k=1}^{\infty}\left[\left(\left[\frac{w}{m}\right]+1\right) m-w-1\right] \pi(1-\pi)^{k-1} \pi q^{w}(1-q) \\
\quad=\sum_{j=0}^{\infty} \sum_{w=j m}^{(j+1) m-1}[(j+1) m-w-1] \pi q^{w}(1-q) \\
=\pi(1-q) \sum_{j=0}^{\infty} \sum_{w=j m}^{(j+1) m-1}[(j+1) m-w-1] q^{w} \\
=\pi(1-q) \sum_{u=0}^{m-1}\{m-1-u\} q^{u} \sum_{j=0}^{\infty} q^{j m} \\
\quad=\pi(1-q)\left[\frac{(m-1)-[(m-1)-(m-1)] q^{m}}{(1-q)}+\frac{(-1) q\left(1-q^{m-1}\right)}{(1-q)^{2}}\right] \frac{1}{1-q^{m}} \\
\quad=\pi(1-q)\left[\frac{(m-1)}{(1-q)}-\frac{q\left(1-q^{m-1}\right)}{(1-q)^{2}}\right] \frac{1}{1-q^{m}} \\
=\frac{\pi}{1-q^{m}}\left[(m-1)-\frac{q\left(1-q^{m-1}\right)}{(1-q)}\right]
\end{aligned}
$$

e 


$$
\begin{aligned}
\sum_{w=0}^{\infty} \sum_{k=1}^{\infty}( & k-1)(m-1) \pi(1-\pi)^{k-1} \pi q^{w}(1-q) \\
= & (m-1) \pi \sum_{k=1}^{\infty}(k-1)(1-\pi)^{k-1} \pi \\
= & (m-1) \pi \frac{(1-\pi)}{\pi},
\end{aligned}
$$

substituindo (3.12) e (3.13) em (3.11) obtêm-se

$$
\begin{aligned}
E\left(\sum_{i=1}^{m \xi+l} X_{i}\right) & =E\left[E\left(\sum_{i=1}^{m \xi+l} X_{i} \mid \xi, W\right)\right] \\
& =\frac{\pi}{1-q^{m}}\left[(m-1)-\frac{q\left(1-q^{m-1}\right)}{(1-q)}\right]+(m-1) \pi \frac{(1-\pi)}{\pi}+l \pi+[\pi+(1-\pi)] \\
& =\frac{\pi}{1-q^{m}}\left[m-\frac{1-q^{m}}{(1-q)}\right]+(m-1)(1-\pi)+l \pi+\pi+(1-\pi) \\
& =\left[\frac{m}{1-q^{m}}-\frac{1}{(1-q)}+1\right] \pi+m(1-\pi)+l \pi \\
& =\left[\frac{m}{1-q^{m}}-\frac{q}{(1-q)}\right] \pi+m(1-\pi)+l \pi \\
& =\left[m+\frac{m q^{m}}{1-q^{m}}-\frac{q}{(1-q)}\right] \pi+m(1-\pi)+l \pi .
\end{aligned}
$$

A expressão (3.14) coincide com o coeficiente de $C_{D}$ obtido por Nayebpour and Woodall (1993: pg. 58, expressão 4.5a). Substituindo (3.14) e (3.6) em (3.9) obtêm-se

$$
E(C)=\left(\left[m+\frac{m q^{m}}{1-q^{m}}-\frac{q}{(1-q)}\right] \pi+m(1-\pi)+l \pi\right) C_{D}+\left(\frac{1-\pi}{\pi}+\frac{1}{1-q^{m}}\right) C_{i}+C_{a}
$$

No entanto, o coeficiente de $C_{i}$ na expressão do custo esperado do procedimento de controle em um ciclo, apresentado por Nayebpour and Woodall (1993: pg. 58, expressão 4.5a), não coincide com o coeficiente de $C_{i}$ em (3.15). A diferença ocorre pois a expressão obtida por Nayebpour and Woodall não considera a interrupção das inspeções durante o atraso. Isto justifica o acréscimo de $\left[\frac{l}{m}\right]$ no coeficiente da expressão de Nayebpour and Woodall. 
Para a situação em que a inspeção retrospectiva (100\%) é realizada durante o último intervalo de diagnóstico mais o período de atraso, são considerados os fatores de custo $C_{i}, C_{a}$, $C_{d}$ e $C_{D}$. Neste caso, $C_{i}$ e $C_{a}$ são definidos da mesma forma que na situação anterior, $C_{d}$ é o custo de um item não conforme identificado por inspeção retrospectiva e $C_{D}$ o custo de um item não conforme não identificado por inspeção retrospectiva e enviado para processos posteriores ou para o mercado. Assim, o custo do procedimento de controle em um ciclo pode ser expresso por

$$
C=C_{D} \sum_{j=0}^{m(\xi-1)} X_{j}+C_{d} \sum_{j=m(\xi-1)+1}^{m \xi+l} X_{j}+C_{i}(\xi+l+m-1)+C_{a}
$$

Consequentemente,

$$
\begin{aligned}
E(C) & =C_{D} E\left(\sum_{j=0}^{m(\xi-1)} X_{j}\right)+C_{d} E\left(\sum_{j=m(\xi-1)+1}^{m \xi+l} X_{j}\right)+C_{i}(E(\xi)+l+m-1)+C_{a} \\
& =C_{D} E\left[E\left(\sum_{j=0}^{m(\xi-1)} X_{j} \mid \xi, W\right)\right]+C_{d} E\left[E\left(\sum_{j=m(\xi-1)+1}^{m \xi+l} X_{j} \mid \xi, W\right)\right]+C_{i}(E(\xi)+l+m-1)+C_{a} .
\end{aligned}
$$

Como

$$
\begin{aligned}
& E\left(\sum_{j=0}^{m(\xi-1)} X_{j} \mid W\right.\left.=w, \xi=\left[\frac{w}{m}\right]+k\right) \\
&=0, \quad \text { se } k=1 \\
&=\left[\left(\left[\frac{w}{m}\right]+1\right) m-w-1\right] \pi+(k-2)(m-1) \pi, \text { se } k>1, \\
& E\left(\sum_{j=0}^{m(\xi-1)} X_{j}\right)=\sum_{w=0}^{\infty} \sum_{k=2}^{\infty}\left\{\left[\left(\left[\frac{w}{m}\right]+1\right) m-w-1\right] \pi+(k-2)(m-1) \pi\right\} q^{w}(1-q) \pi(1-\pi)^{k-1} .
\end{aligned}
$$

Por outro lado, 


$$
\begin{aligned}
\sum_{w=0}^{\infty} \sum_{k=2}^{\infty}(k-2)(m-1) \pi q^{w} & (1-q) \pi(1-\pi)^{k-1}= \\
& =(m-1) \pi \sum_{w=0}^{\infty} q^{w}(1-q) \sum_{k=2}^{\infty}(k-2) \pi(1-\pi)^{k-1} \\
& =(m-1) \pi\left[\frac{1}{\pi}-\pi-2(1-\pi)\right] \\
& =(m-1) \pi\left[\frac{1}{\pi}+\pi-2\right] \\
& =(m-1)(1-\pi)^{2}
\end{aligned}
$$

e

$$
\begin{aligned}
\sum_{w=0}^{\infty} \sum_{k=2}^{\infty}\left[\left(\left[\frac{w}{m}\right]+1\right)\right. & m \bar{x} w-1] \pi q^{w}(1-q) \pi(1-\pi)^{k-1} \\
& =\sum_{w=0}^{\infty}\left[\left(\left[\frac{w}{m}\right]+1\right) m-w-1\right] \pi q^{w}(1-q)(1-\pi) \\
& =\pi(1-\pi)(1-q) \sum_{j=0}^{\infty} \sum_{w=j m}^{(j+1) m-1}\{(j+1) m-1-w\} q^{w} \\
& =\pi(1-\pi)(1-q) \sum_{j=0}^{\infty} \sum_{u=0}^{m-1}(m-1-u) q^{j m+u} \\
& =\pi(1-\pi)(1-q) \sum_{j=0}^{\infty} q^{j m}\left[\frac{(m-1)-[(m-1)-(m-1)] q^{m}}{1-q}+\frac{(-1) q\left(1-q^{m-1}\right)}{(1-q)^{2}}\right] \\
& =\pi(1-\pi)(1-q) \sum_{j=0}^{\infty} q^{j m}\left[\frac{m-1}{1-q}-\frac{q\left(1-q^{m-1}\right)}{(1-q)^{2}}\right] \\
& =\pi(1-\pi)\left[m-\frac{1-q^{m}}{1-q}\right] \frac{1}{1-q^{m}} .
\end{aligned}
$$

Substituindo (3.20) e (3.21) em (3.19) obtemos o coeficiente de $C_{D}$ na expressão (3.17), isto é

$$
\begin{aligned}
E\left(\sum_{j=0}^{m(\xi-1)} X_{j}\right) & =\pi(1-\pi)\left[m-\frac{1-q^{m}}{1-q}\right] \frac{1}{1-q^{m}}+(m-1)(1-\pi)^{2} \\
& =\pi(1-\pi)\left[\frac{m}{1-q^{m}}-\frac{1}{1-q}\right]+(m-1)(1-\pi)^{2} .
\end{aligned}
$$


Observe que

$$
\begin{aligned}
\pi(1-\pi)\left[\frac{m}{1-q^{m}}\right. & \left.-\frac{1}{1-q}\right]+(m-1)(1-\pi)^{2} \\
& =\pi(1-\pi)\left[\frac{m}{1-q^{m}}-\frac{q}{1-q}\right]+(m-1)(1-\pi)^{2}-\pi(1-\pi) \\
& =\pi(1-\pi)\left[\frac{m}{1-q^{m}}-\frac{q}{1-q}\right]+m(1-\pi)^{2}-(1-\pi)^{2}-\pi(1-\pi) \\
& =\pi(1-\pi)\left[\frac{m}{1-q^{m}}-\frac{q}{1-q}\right]+m(1-\pi)^{2}-(1-\pi)
\end{aligned}
$$

coincide com o coeficiente de $C_{D}$ obtido por Nayebpour and Woodall (1993: pg 58, expressão 4.2) a menos do fator $-(1-\pi)$. Este erro, como mencionamos, havia sido apontado anteriormente por Borges et al. (1999).

Da mesma forma

$$
\begin{aligned}
E\left(\sum_{j=m(\xi-1)+1}^{m \overline{\xi+l} X_{j} \mid W}\right. & \left.=w, \xi=\left[\frac{w}{m}\right]+k\right) \\
& =\left[\left(\left[\frac{w}{m}\right]+1\right) m-w-1\right] \pi+l \pi+1, \text { se } k=1 \\
& =(m-1) \pi+l \pi+1, \quad \text { caso contrário. }
\end{aligned}
$$

Assim,

$$
\begin{aligned}
& E\left(\sum_{j=m(\xi-1)+1}^{m \xi+l} X_{j}\right)=\sum_{w=0}^{\infty}\left\{\left[\left(\left[\frac{w}{m}\right]+1\right) m-w-1\right] \pi+l \pi+1\right\} q^{w}(1-q) \pi+ \\
& \quad+\sum_{\mathrm{w}=0}^{\infty} \sum_{\mathrm{k}=2}^{\infty}\{(m-1) \pi+l \pi+1\} q^{w}(1-q) \pi(1-\pi)^{k-1} \\
& \quad=\pi^{2}(1-q) \sum_{w=0}^{\infty}\left[\left(\left[\frac{w}{m}\right]+1\right) m-w-1\right] q^{w}+l \pi^{2}+\pi+\{(m-1) \pi+l \pi+1\}(1-\pi) \\
& \quad=\pi^{2}(1-q)\left[\frac{m-1}{1-q}-\frac{q\left(1-q^{m-1}\right)}{(1-q)^{2}}\right] \frac{1}{1-q^{m}}+l \pi^{2}+\pi+ \\
& \quad+(m-1) \pi(1-\pi)+l \pi(1-\pi)+(1-\pi) \\
& =\pi^{2}\left[\frac{m}{1-q^{m}}-\frac{1}{1-q}\right]+l \pi^{2}+(m-1) \pi(1-\pi)+l \pi(1-\pi)+1
\end{aligned}
$$


Observe novamente que

$$
\begin{aligned}
\pi^{2}\left[\frac{m}{1-q^{m}}-\frac{1}{1-q}\right]+l \pi^{2}+(m-1) \pi(1-\pi)+l \pi(1-\pi)+1 \\
=\pi^{2}\left[\frac{m}{1-q^{m}}-\frac{1}{1-q}\right]+l\left(\pi^{2}+\pi(1-\pi)\right)+(m-1) \pi(1-\pi)+1 \\
=\pi^{2}\left[\frac{m}{1-q^{m}}-\frac{q}{1-q}\right]+l \pi+(m-1) \pi(1-\pi)+1-\pi^{2} \\
=\pi^{2}\left[\frac{m}{1-q^{m}}-\frac{q}{1-q}\right]+l \pi+m \pi(1-\pi)-\pi(1-\pi)+1-\pi^{2} \\
=\pi^{2}\left[\frac{m}{1-q^{m}}-\frac{q}{1-q}\right]+l \pi+m \pi(1-\pi)-\pi+\pi^{2}+1 \\
=\pi^{2}\left[\frac{m}{1-q^{m}}-\frac{q}{1-q}\right]+l \pi+m \pi(1-\pi)+(1-\pi)
\end{aligned}
$$

coincide com o coeficiente de $C_{d}$ em 4.2 dado por Nayebpour and Woodall(1993: pg 58, expressão 4.2) a menos do fator (1- $\pi$ ). A soma dos coeficientes de $C_{D}$ e $C_{d}$ em (3.26) e (3.23), entretanto, coincide com o coeficiente de $C_{D}$ em (3.15) no caso em que não há inspeção retrospectiva, como era de se esperar.

Substituindo (3.6), (3.22) e (3.25) em (3.17) obtêm-se

$$
\begin{aligned}
E(C)= & \left(\left(\frac{m}{1-q^{m}}-\frac{q}{1-q}\right) \pi^{2}+m \pi(1-\pi)+l \pi+(1-\pi)\right) C_{d}+ \\
& \left(\left(\frac{m}{1-q^{m}}-\frac{1}{1-q}\right) \pi(1-\pi)+m(1-\pi)^{2}-(1-\pi)\right) C_{D}+ \\
& \left(\frac{1-\pi}{\pi}+\frac{1}{1-q^{m}}+l+m-1\right) C_{i}+C_{a} .
\end{aligned}
$$

Observe que o coeficiente de $C_{i}$ na expressão do custo esperado do procedimento de controle em um ciclo, apresentado por Nayebpour and Woodall (1993: pg. 58, expressão 4.5a) também não coincide com o coeficiente de $C_{i}$ em (3.15). A diferença ocorre pois Nayebpour and Woodall não consideram a interrupção das inspeções durante o atraso e incluem o termo $(m+l)$ no custo de $C_{i}$. 
A partir das expressões analíticas para $E(C)$ e $E(T)$, desenvolvidas acima, a obtenção do intervalo ótimo de diagnóstico se torna uma questão apenas computacional. Devido a complexidade da expressão analítica para o custo médio assintótico do procedimento, por item produzido, o valor $m$ que minimiza (3.1) deve ser obtido, como fazem Nayebpour and Woodall (1993) e Borges et al. (2000), por busca direta. Programas computacionais como o Mathematica ${ }^{\circledR}$, por exemplo, facilitam sobremaneira esta tarefa.

Para concluir vamos examinar as questões da estimação dos parâmetros $q$ e $\pi$ do processo e da estimação do ponto de mudança $W$. Esta última, em particular, tem uma certa afinidade com a recomendação técnica de inspecionar retrospectivamente, em cada ciclo, apenas os itens produzidos durante o último intervalo de diagnóstico e o atraso, feita por Nayebpour and Woodall (1993). Observe que uma estimativa do ponto de mudança $W$ nos permitiria inspecionar retrospectivamente até o ponto $W$, ou descartar essas unidades conforme os custos envolvidos. Examinaremos essas questões sob o princípio de máximaverossimilhança.

Para determinar o estimador do ponto de mudança do processo, vamos supor que não é realizada inspeção retrospectiva e que o valor $\pi$ é conhecido. Não é difícil ver que a função de verossimilhança neste caso é dada por

$$
\begin{array}{rlrl}
L(w)=P\{\xi=k \mid W=w\} & =0, & & \text { se }\left[\frac{w}{m}\right] \geq k \\
& =\pi(1-\pi)^{k-\left[\frac{w}{m}\right]-1} & \text { se }\left[\frac{w}{m}\right]<k
\end{array}
$$

Considerando $\left[\frac{w}{m}\right]=j$, temos

$$
\begin{array}{ccc}
L(w)=P\{\xi=k \mid W=w\}=\pi(1-\pi)^{k-j-1} & \text { se } j m \leq w<(j+1) m, j<k \\
=0 & \text { se } j m \leq w<(j+1) m, j \geq k
\end{array}
$$

Assim, $L(w)$ é máximo se $(k-1) m \leq w<k m$ e a estimativa de máxima verossimilhança não é única. Este fato, portanto, justifica informalmente a recomendação de inspecionar retrospectivamente as unidades produzidas durante o último intervalo de inspeção e o atraso. 
Se $\pi$ não for conhecido, é necessário obtermos pelo menos uma estimativa de seu valor.

Para obtermos, simultaneamente, uma estimativa de $w$ e $\pi$, observe que

$$
\log L(\pi)=\log \pi+(k-j-1) \log (1-\pi)
$$

para $j m \leq w<(j+1) m$. Como

$$
\frac{\partial \log L(\pi)}{\partial \pi}=\frac{1}{\hat{\pi}}-(k-j-1) \frac{1}{(1-\hat{\pi})}=0
$$

implica

$$
(1-\hat{\pi})=(k-j-1) \hat{\pi}
$$

e portanto

$$
\hat{\pi}=\frac{1}{k-j}
$$

Um estimador de máxima verossimilhança de $w$ e $\pi$ seria $(\xi-1) m$ e 1 . Observe que, já que não estamos fazendo inspeção retrospectiva, estimamos $\pi$ essencialmente com base em um sucesso obtido em um ensaio. A estimação de $\pi$ pode ser melhorada quando houver inspeção retrospectiva. Neste caso, teremos observado também dados referentes à fração defeituosa durante o último intervalo de diagnóstico e o atraso.

A estimativa simultânea de $q$ e $\pi$ pode ainda ser obtida por máxima verossimilhança se observarmos que

$$
\begin{aligned}
P\{\xi=k \mid W=w\} & =0 & & \text { se } j m \leq w<(j+1) m \text { e } k \leq j \\
& =\pi(1-\pi)^{k-j-1} & & \text { se } j m \leq w<(j+1) m \text { e } k>j
\end{aligned}
$$

e, consequentemente, que 


$$
\begin{aligned}
P\{\xi=k\} & =\sum_{w=0}^{\infty} P\{\xi=k \mid W=w\} q^{w}(1-q) \\
& =\sum_{j=0}^{\infty} \sum_{w=j m}^{(j+1) m-1} P\{\xi=k \mid W=w\} q^{w}(1-q) \\
& =\sum_{j=0}^{k-1} \sum_{w=j m}^{(j+1) m-1}(1-\pi)^{k-j-1} \pi q^{w}(1-q) \\
& =\pi(1-q) \sum_{j=0}^{k-1}(1-\pi)^{k-j-1} \sum_{w=j m}^{(j+1) m-1} q^{w} \\
& =\pi(1-q) \sum_{j=0}^{k-1}(1-\pi)^{k-j-1} q^{j m} \frac{1-q^{m}}{1-q} \\
& =\pi\left(1-q^{m}\right)(1-\pi)^{k-1} \frac{\sum_{j=0}^{k-1}\left(\frac{q^{m}}{1-\pi}\right)^{j}}{1-\left(q^{k m} /(1-\pi)^{k}\right)} \\
& =\pi\left(1-q^{m}\right)(1-\pi)^{k-1} \frac{1-\left(q^{m} /(1-\pi)\right)}{(1-\pi)^{k}\left[1-\pi-q^{m}\right]} \\
& =\pi\left(1-q^{m}\right)(1-\pi)^{k-1} \frac{\left[(1-\pi)^{k}-q^{k m}\right](1-\pi)}{(1-\pi} \\
& =\pi\left(1-q^{m}\right) \frac{(1-\pi)^{k}-q^{k m}}{(1-\pi)}
\end{aligned}
$$

Reparametrizando para $\theta=1-\pi$ e $u=q^{m}$, a função de verossimilhança será dada por

$$
L(\theta, u)=(1-\theta)(1-u) \frac{\theta^{k}-u^{k}}{\theta-u}
$$

Assim, estimativas de máxima verossimilhança para $\theta$ e $u$ podem ser obtidas resolvendo-se, numericamente o sistema não-linear

$$
\begin{aligned}
& \frac{\partial L(\theta, u)}{\partial \theta}=0 \\
& \frac{\partial L(\theta, u)}{\partial u}=0 .
\end{aligned}
$$




\section{Conclusão}

A abordagem de Taguchi para monitorar processos por atributos é vantajosa por sua simplicidade. Entretanto, as expressões desenvolvidas por Taguchi e seus colaboradores para o custo médio assintótico do procedimento de controle por item produzido e os intervalos ótimos de diagnóstico correspondentes não são corretas. Para determiná-las, Taguchi não toma como base em um modelo probabilístico formal e estabelece seus resultados com base em aproximações e suposições não fundamentadas.

Para corrigir os defeitos dos trabalhos originais de Taguchi e seus colaboradores, foi apresentado um modelo probabilístico unificado para descrever um processo de produção submetido a monitoramento de Taguchi para atributos e obtêm-se, a partir dele, a expressão para o custo médio assintótico do procedimento de controle, por item produzido. Esse modelo é utilizado para corrigir um erro encontrado em um dos resultados obtidos por Nayebpour e Woodall (1993) no desenvolvimento de um modelo alternativo com a mesma finalidade.

A questão da estimação dos parâmetros do processo a partir de dados observados no procedimento de controle, com particular ênfase no ponto de mudança ou instante da falha do processo, também foi abordada. Entretanto, para obter as estimativas de máxima verossimilhança de $\theta$ e $u$ deve-se resolver um sistema não-linear que possui parâmetros não identificáveis. Pesquisas futuras devem ser realizadas com o objetivo de solucioná-lo. 


\section{Referências Bibliográficas}

[1] Berry, S.A., (1974). Techniques in the Application of Computers to Industrial Monitoring, in CAD/CAM and the Computer Revolution, Society of Manufacturing Engineers, 221-39.

[2] Borges, W., Ho L. L. and Turnes O., (2000). An Analysis of Taguchi's on-Line Quality Monitoring Procedure for Attributes with Diagnosis Eros, Technical Report, IME-USP.

[3] Burr, I. W., (1953). Engineering Statistics and Quality Control, New York: McGraw-Hill.

[4] Chiu, W. K., (1975). Economic Design of Attribute Control Charts, Technometrics, 17, 817.

[5] Chiu, W. K., (1976). Economic Design of $n p$ Charts for Processes Subject to a Multiplicity of Assignable Causes, Management Science, 23, 404-11.

[6] Cowden, D. J., (1957). Statistical Methods in Quality Control, Englewood Cliffs, NJ: Prentice-Hall.

[7] Duncan, A. J., (1956). The Economic Design of $\bar{X}$ Charts Used to Maintain Current Control of a Process, Journal of the American Statistical Association, 66, 107-21.

[8] Duncan, A. J., (1978). The Economic Design of $p$-Charts to Maintain Current Control of a Process: Some Numerical Results, Technometrics, Vol. 20, No. 3, pp. 235-43.

[9] Duncan, A. J., (1986). Quality Control and Industrial Statistics, $5^{\text {th }}$ ed., Irwin, Homewood, III.

[10] Feigenbaum, A.V., (1961). Total Quality Control, New York: McGraw-Hill.

[11] Gibra, I. N., (1978). Economically Optimal Determination of the Parameters of $n p$ Control Charts, Journal of Quality Technology, Vol. 10, No. 1, pp. 12-9.

[12] Grant, E. L., and Leavenworth, R. S., (1980). Statistical Quality Control, New York: McGraw-Hill. 
[13] Ishikawa, K., (1976). Guide to Quality Control, Tokyo: Asian Productivity Organization.

[14] Juran, J. M., Gryna, F. M., Jr., and Bingham, R. S., Jr., (1974). Quality Control Handbook, New York: McGraw-Hill.

[15] Landany, S. P. (1973), Optimal Use of Control Charts Controlling Current Production, Journal of Quality Technology, 10, 12-9.

[16] Montgomery, D. C. and Heikes, R. G., and Mance, J. F., (1975). Economic Design of Fraction Defective Control Charts, Management Science, Vol. 21, No. 11, pp.1272-84.

[17] Montgomery, D. C. and Heikes, R. G., (1976). Process Failure Mechanisms and Optimal Design of Fraction Defective Control Charts, AIIE Transactions, Vol. 8, No. 4 pp. 467-72.

[18] Montgomery, D C., (1996). Introduction to Statistical Quality Control, $3^{\text {rd }}$ Edition, John Wiley and Sons, New York.

[19] Nayepour, M. R., (1991). An Analysis of Taguchi's On-line Quality Monitoring Procedures for Attribute Characteristics, Technical Report, University of Houston - Clear Lake, Dept. of Mathematical Science.

[20] Nayepour, M. R. and Woodall, W. H., (1993). An Analysis of Taguchi's On-line Quality Monitoring Procedure for Attributes, Technometrics, 35, 53-60.

[21] Nelson, L. S., (1984). The Shewart Control Chart - Test for Special Causes, Journal of Quality Technology, Vol 16.

[22] Taguchi, G., (1981). On-line Quality Control During Production, Japanese Standards Association, Tokyo.

[23] Taguchi, G., (1984). Quality Evaluation for Quality Assurance, American Supplier Institute, Dearborn, MI.

[24] Taguchi, G., (1985). Quality Engineering in Japan, Communications in Statistics Theory ans Methods, 14, 2785-801.

[25] Taguchi, G., Elsayed, E. A. and Hsiang, T (1989). Quality Engineering in Production Systems, McGraw-Hill, New York, NY.

[26] Western Eletric, (1956). Statistical Quality Control Handbook, Western Eletric Corporation, Indianapolis, Ind.

[27] Willians, W. W., Looney, S. W. and Peters, M. H., (1985). Use of Curtailed Sampling Plans in the Economic Design of $n p$-Control Charts, Technometrics, 27, 57-63. 